\title{
The viscosity of a dilute suspension of rough spheres
}

\author{
By HELEN J. WILSON and ROBERT H. DAVIS $\dagger$ \\ Department of Chemical Engineering, University of Colorado, Boulder, CO 80309-0424, USA
}

(Received 1999 - in revised form 2000)

We consider the flow of a dilute suspension of equisized solid spheres in a viscous fluid. The viscosity of such a suspension is dependent on the volume fraction, $c$, of solid particles. If the particles are perfectly smooth, then solid spheres will not come into contact, because lubrication forces resist their approach. In this paper, however, we consider particles with microscopic surface asperities such that they are able to make contact. For straining motions we calculate the $O\left(c^{2}\right)$ coefficient of the resultant viscosity, due to pairwise interactions. For shearing motions (for which the viscosity is undetermined because of closed orbits on which the probability distribution is unknown) we calculate the $c^{2}$ contribution to the normal stresses $N_{1}$ and $N_{2}$. The viscosity in strain is shown to be slightly lower than that for perfectly smooth spheres, though the increase in the $O(c)$ term caused by the increased effective radius due to surface asperities will counteract this decrease. The viscosity increases with increasing contact friction coefficient. The normal stresses $N_{1}$ and $N_{2}$ are zero if the surface roughness height is less than a critical value of $2.11 \times 10^{-4}$ times the particle radius, and then become negative as the roughness height is increased above this value. $N_{1}$ is larger in magnitude than $N_{2}$.

This file is not a faithful reproduction of the paper printed in JFM; rather, errors which were discovered after publication have been corrected here in red.

J. Fluid Mech. (2000), vol. 421, pp. 339-367. DOI: 10.1017/S0022112000001695

\section{Introduction}

The study of suspensions of small particles has been of interest to scientists for many years. When the particles are small enough that the suspending fluid may be assumed to have no inertia, but not so small that Brownian motion need be taken into account, particular progress for dilute suspensions may be made without recourse to large-scale simulations.

In this paper we study dilute suspensions of rough spherical particles in a Newtonian fluid. It is well known (Einstein 1906, 1911) that a very dilute suspension of spheres, whether rough or smooth (provided the roughness is small compared to the particle radius), behaves to first order in the small volume fraction $c$ as a Newtonian fluid with effective viscosity $\mu(1+5 c / 2)$, where $\mu$ is the viscosity of the suspending fluid.

The corresponding calculation at order $c^{2}$ is more difficult. For perfectly smooth spheres, Batchelor \& Green $(1972 a, b)$ calculated the stresses acting in particular flows, but the rheology of the fluid depends on the history of the bulk flow and cannot be simply expressed for all flows. For example, in simple shear flow two particles may rotate endlessly around one another, causing a viscosity which is periodic in time. In axisymmetric

$\dagger$ To whom correspondence should be addressed (robert.davis@colorado.edu, 303-492-7314 phone, 303-492-4341 fax). 
straining flows, on the other hand, the $O\left(c^{2}\right)$ term of the viscosity is known in terms of mobility functions, which we define later, and the total or effective viscosity for equisized smooth spheres is

$$
\mu\left(1+\frac{5}{2} c+\left[\frac{5}{2}+\frac{15}{2} \int_{2}^{\infty} J(s) q(s) s^{2} \mathrm{~d} s\right] c^{2}+O\left(c^{3}\right)\right) .
$$

Because the calculation at $O\left(c^{2}\right)$ involves the interactions between pairs of particles, the issue of microscopic particle roughness becomes important. It has been observed (Batchelor \& Green $1972 a$, p. 417),

"... that in practice there may be departures from the theoretical formulae due to small surface irregularities..."

Perfectly smooth spheres subject to finite forces in a continuum fluid can never come into contact because of the lubrication forces between them. However, experiments (Arp \& Mason 1977; Zeng, Kerns \& Davis 1996) have shown conclusively that, for real particles which appear smooth to the naked eye, microscopic surface asperities can cause interparticle contacts. These contacts break the reversibility condition which is a property of Stokes flow, and can lead to an empty wake behind each particle in some flows. The contacts can also (by conservation of particles) lead to surfaces, fixed relative to one particle, on which there is a high probability of finding a second particle. These two microstructural effects are expected to have repercussions for the rheology of the suspension containing rough particles.

The rheology at order $c^{2}$ will depend on the model chosen to describe the surface asperities of the particles and the contact between them. There are three models in common use (see, for example, da Cunha \& Hinch 1996; Davis 1992): hard-sphere repulsion, stick-rotate and roll-slip. Hard-sphere repulsion is a special case of the roll-slip model, and experimental results shown by Zeng et al. (1996) suggest that the roll-slip model is more realistic than the stick-rotate model. Thus, in this paper we use the roll-slip friction model (including the frictionless limit of hard-sphere repulsion) to investigate the rheology of a dilute suspension of rough particles.

In $\S 2$ we pose the problem rigorously, and solve it as far as is possible for a general imposed flow field. In $\S 3$ we complete the calculation for axisymmetric straining motions, and in $\S 4$ for shear flows. Concluding remarks are given in $\S 5$.

\section{Formulation of the problem}

We consider a Newtonian fluid of viscosity $\mu$, containing neutrally buoyant suspended solid spherical particles of radius $a$ at volume fraction $c$. The particles are force- and torque-free on a macroscopic level, which is to say the only forces (other than hydrodynamic forces) acting on individual particles are short-range and symmetrical. We allow for contact forces between the particles, which lead to no net force acting on the system as a whole.

When two particles come into contact, they behave according to the roll-slip model of Davis (1992). At an interparticle surface-surface separation $h_{c}=a \zeta$, with $\zeta \ll 1$, their approach is halted by small surface asperities. They remain in contact (with the minimum gap between their nominal surfaces equal to $h_{c}$ ) for as long as the net hydrodynamic forces acting on them are compressive. Once the hydrodynamic forces act to separate the spheres, the contact breaks and there is no contact force; the particles separate unhindered except by hydrodynamic forces. While the particles are in contact, the normal contact force (the contact force acting parallel to the line of centres) on each sphere is equal and opposite to the normal hydrodynamic force on that sphere. The in- 
teraction forces in a direction tangent to the contacting surfaces are then assumed to be a combination of hydrodynamic forces unaffected by surface roughness and contact, and a frictional contact force. The tangential friction force depends both on the hydrodynamic forces and on a coefficient of friction, $\nu$. Essentially, if the magnitude of the normal force is large enough, then the particles roll around one another with the frictional force balancing the hydrodynamic force at contact. Otherwise a frictional force (of magnitude $\nu$ times the magnitude of the normal force) is exerted to oppose the relative tangential motion, and the particles slip around each other. In the limit $\nu=0$, the contact force only has a normal component and is just a hard-sphere repulsion. This model has two dimensionless parameters, $\nu$ and $\zeta$, with typical physical values (Smart \& Leighton 1989) of $10^{-3}<\zeta<10^{-2}$ and (Zeng et al. 1996) $0.1<\nu<0.4$.

The detailed description of the problem (with smooth particles) can be found in Batchelor (1967), pp. 246-253. Here we present only a shortened version.

The far-field velocity is imposed as the linear function

$$
\boldsymbol{U}^{\infty}=\boldsymbol{\Omega} \times \boldsymbol{x}+E \cdot \boldsymbol{x},
$$

where $E_{i j}$ is a traceless and symmetric tensor. The suspension takes on this velocity only in an average sense, as the presence of rigid particles and the interactions between them affect the local flow.

The stress tensor at any point in the ambient fluid (with Newtonian viscosity $\mu$ ) is given by

$$
\Sigma_{i j}=-p \delta_{i j}+2 \mu E_{i j}+\Sigma_{i j}^{(p)},
$$

where the particle stress (deriving from the rigidity of a particle in its interaction with the surrounding suspension, and from interparticle forces) is summed over all particles. The isotropic term is the pressure in the fluid, which is perturbed by the presence of the particles (Brady 1993). Since the fluid is incompressible, however, this pressure may be determined only up to an arbitrary constant and has no effect on the flow. We choose not to investigate here the perturbation to it caused by the presence of the particles. We expand the extra (particle) stress in powers of the small volume concentration, $c$, while averaging over the volume of the suspension. The leading-order term (which is $O(c)$ ) is derived from consideration of the extra dissipation caused by an isolated sphere in the far-field flow $\boldsymbol{U}^{\infty}$, and the $O\left(c^{2}\right)$ term from binary interactions between pairs of particles. Following the work of Zinchenko (1984), we can express the extra stress as

$$
\begin{aligned}
\boldsymbol{\Sigma}^{(p)}=5 c \mu E+5 c^{2} \mu E & \\
+\frac{15 c^{2} \mu}{4 \pi a^{3}} \int_{r \geq 2 a} & {\left[\left(\frac{S^{h}\left(\boldsymbol{x}_{0}, \boldsymbol{x}_{0}+\boldsymbol{r}\right)}{(20 / 3) \pi \mu a^{3}}-E\right) p(\boldsymbol{r})-e\left(\boldsymbol{x}_{0}, \boldsymbol{x}_{0}+\boldsymbol{r}\right)\right] \mathrm{d} \boldsymbol{r} } \\
& \quad+\frac{9 c^{2}}{32 \pi^{2} a^{6}} \int_{\text {contact }} S^{c}\left(\boldsymbol{x}_{0}, \boldsymbol{x}_{0}+\boldsymbol{r}\right) p(\boldsymbol{r}) \mathrm{d} \boldsymbol{r}+O\left(c^{3}\right),
\end{aligned}
$$

in which $\boldsymbol{n}=\boldsymbol{r} / r$,

$$
\begin{aligned}
S^{h}=\frac{20}{3} \pi a^{3} \mu\{(1+K(s)) E+[(E \cdot \boldsymbol{n}) \boldsymbol{n} & +\boldsymbol{n}(E \cdot \boldsymbol{n})] L(s) \\
& \left.+(\boldsymbol{n} \cdot E \cdot \boldsymbol{n})\left[\boldsymbol{n n} M(s)-\left(\frac{2}{3} L(s)+\frac{1}{3} M(s)\right) I\right]\right\}
\end{aligned}
$$

is the stresslet due to the rigidity of one particle at $\boldsymbol{x}_{0}$ in the presence of a second particle at $\boldsymbol{x}_{0}+\boldsymbol{r}$, with $s=r / a$, and $p(\boldsymbol{r})$ is the pair distribution function: the probability of finding a particle centred at $\boldsymbol{x}_{0}+\boldsymbol{r}$ given that there is a particle centred at $\boldsymbol{x}_{0}$ (normalised so that $p(\boldsymbol{r}) \rightarrow 1$ as $r \rightarrow \infty)$. The term $e\left(\boldsymbol{x}_{0}, \boldsymbol{x}_{0}+\boldsymbol{r}\right)$ is the perturbation to the rate- 
of-strain tensor at $\boldsymbol{x}_{0}$ caused by a single particle centred at $\boldsymbol{x}_{0}+\boldsymbol{r}$. It was devised by Batchelor \& Green (1972a) to make the stress integral uniformly convergent (so that it is valid to perform the integrations in any order), and if the angle integrals are carried out first it does not contribute to the stress. Thus, if the angle integrals are always performed before the radial integral, we have

$$
\begin{aligned}
\boldsymbol{\Sigma}^{(p)}=5 c \mu E+5 c^{2} \mu & +\frac{9 c^{2}}{16 \pi^{2} a^{6}} \int_{\text {contact }} S^{c}\left(\boldsymbol{x}_{0}, \boldsymbol{x}_{0}+\boldsymbol{r}\right) p(\boldsymbol{r}) \mathrm{d} \boldsymbol{r} \\
+ & \frac{15 c^{2} \mu}{4 \pi a^{3}} \int_{r \geq 2 a}[K(s) E+[(E \cdot \boldsymbol{n}) \boldsymbol{n}+\boldsymbol{n}(E \cdot \boldsymbol{n})] L(s) \\
& \left.+(\boldsymbol{n} \cdot E \cdot \boldsymbol{n})\left[\boldsymbol{n n} M(s)-\left(\frac{2}{3} L(s)+\frac{1}{3} M(s)\right) I\right]\right] p(\boldsymbol{r}) \mathrm{d} \boldsymbol{r}+O\left(c^{3}\right),
\end{aligned}
$$

The unfamiliar third term (derived in appendix B), ignoring the isotropic part, is

$$
\begin{aligned}
S^{c}\left(\boldsymbol{x}_{0}, \boldsymbol{x}_{0}+\boldsymbol{r}\right)=\frac{1}{2} a s[1-A(s)]\left(\boldsymbol{F}_{c} \cdot \boldsymbol{n}\right)\left(\boldsymbol{n n}-\frac{1}{3} l\right) \\
+\frac{1}{4} a s\left[1-B(s)-2\left(y_{11}^{h}+y_{12}^{h}\right)\right]\left(\boldsymbol{F}_{c} \boldsymbol{n}+\boldsymbol{n} \boldsymbol{F}_{c}-2 \boldsymbol{n n}\left(\boldsymbol{F}_{c} \cdot \boldsymbol{n}\right)\right) .
\end{aligned}
$$

The coefficient outside the integral of the force dipole is simply $n^{2}$, where $n$ is the number density $3 c / 4 \pi a^{3}$. The hydrodynamic functions $A, B, J, K, L$ and $M$, as well as $x_{\alpha \beta}^{g}, y_{\alpha \beta}^{g}$ and $y_{\alpha \beta}^{h}$, have been thoroughly investigated in previous work (see, for example, Kim \& Karrila 1991).

Before we can make further progress in identifying the pair-distribution function (the major piece of missing information from the formulation above), we need to find the relative velocities of two particles at specific relative positions, and the force acting between them if they are in contact. In this way we use a trajectory-style analysis to calculate the pair-distribution function. This ability is the major reason why this calculation is easier than the corresponding problem in which Brownian motion is not neglected (see, for example, Brady \& Morris 1997). We consider the interaction between two spheres, as specified above, labelled 1 and 2 . We place particle 1 instantaneously at the origin of the linear flow field $\boldsymbol{U}^{\infty}$ of (2.1), and particle 2 at $\boldsymbol{r}$. The dimensionless centre-to-centre vector is $\boldsymbol{s}=\boldsymbol{r} / a$, with modulus $s$. The particles make contact at $s=s_{c} \equiv 2+\zeta$ (where $\zeta$ is the dimensionless roughness height). Throughout this paper, we denote the value of a mobility function at this separation as $X^{*}=X\left(s=s_{c}\right)$.

\subsection{Velocities}

\subsubsection{Particles not in contact}

We define the mobility functions $A$ and $B$ via the equations governing the motion of the centre of particle 2 relative to the centre of particle 1:

$$
\frac{\mathrm{d} \boldsymbol{r}}{\mathrm{d} t}=\boldsymbol{V}=a s[\boldsymbol{\Omega} \times \boldsymbol{n}+(1-B(s)) E \cdot \boldsymbol{n}+(B(s)-A(s))(\boldsymbol{n} \cdot E \cdot \boldsymbol{n}) \boldsymbol{n}] .
$$

The mobilities $K, L$ and $M$ are defined by the stresslet produced by particle 1 in the presence of particle 2, given by (2.4), and all of the mobility functions are given in Kim \& Karrila (1991).

\subsubsection{Particles in rolling contact}

If we take the fluid velocities on the surface of the particles to be

$$
\begin{aligned}
& \boldsymbol{u}_{1}=\boldsymbol{U}_{1}+\boldsymbol{\omega}_{1} \times \boldsymbol{x}, \\
& \boldsymbol{u}_{2}=\boldsymbol{U}_{2}+\boldsymbol{\omega}_{2} \times(\boldsymbol{x}-a \boldsymbol{s}),
\end{aligned}
$$


then the condition for rolling motion (no relative motion at the point of contact, $\boldsymbol{x}=$ $\left.a s_{c} \boldsymbol{n} / 2\right)$ is

$$
\boldsymbol{U}_{2}=\boldsymbol{U}_{1}+\frac{1}{2} a s_{c}\left(\boldsymbol{\omega}_{1}+\boldsymbol{\omega}_{2}\right) \times \boldsymbol{n} .
$$

The velocities of the two particles may also be derived from the grand mobility matrix formulation (see, for example, Kim \& Karrila 1991). The external flow field and the contact forces and torques acting on each particle are combined to give the velocities. In this case, if the contact force acting on particle 1 is $\boldsymbol{F}_{c}$ then the contact force on particle 2 is $-\boldsymbol{F}_{c}$ and the torques are both $a s_{c} \boldsymbol{n} \times \boldsymbol{F}_{c} / 2$.

Substitution of the resulting forms for the velocities into (2.10) and some manipulation yields the two conditions

$$
\beta_{1}(I-\boldsymbol{n n}) \cdot \boldsymbol{F}_{c}=-\mu a^{2} \beta_{2}(I-\boldsymbol{n n}) \cdot E \cdot \boldsymbol{n}
$$

and

$$
\beta_{3} \boldsymbol{F}_{c} \cdot \boldsymbol{n}=\mu a^{2} s_{c}\left(1-A^{*}\right) \boldsymbol{n} \cdot E \cdot \boldsymbol{n},
$$

where the constants $\beta_{i}$, which derive from the scalar two-sphere mobility functions, are given in Appendix A.

The relative velocity of the two particles is

$$
\boldsymbol{V}^{r}=a s_{c}\left[\boldsymbol{\Omega} \times \boldsymbol{n}+\beta_{4}(I-\boldsymbol{n} \boldsymbol{n}) \cdot E \cdot \boldsymbol{n}\right],
$$

and we can also compute the contact stresslet, using (2.6), and neglecting the term $\left(1-A^{*}\right)^{2} / \beta_{3}$ which is $O(\zeta)$ for solid spheres (and asymptotically small even for liquid drops):

$$
S_{r}^{c}=\mu a^{3} \frac{\beta_{2} \beta_{5}}{4 \beta_{1}}[(I-\boldsymbol{n n}) \cdot E \cdot \boldsymbol{n n}+\boldsymbol{n}(I-\boldsymbol{n n}) \cdot E \cdot \boldsymbol{n}]
$$

\subsubsection{Particles in slipping contact}

For two particles in slipping contact, the normal contact force is the same as it would be for rolling, but the tangential force, while in the same direction, is limited in magnitude by $\nu$ times the magnitude of the normal force:

$$
(I-\boldsymbol{n n}) \cdot \boldsymbol{F}_{c}=-\nu\left(\boldsymbol{n} \cdot \boldsymbol{F}_{c}\right) \frac{(I-\boldsymbol{n n}) \cdot E \cdot \boldsymbol{n}}{|(I-\boldsymbol{n n}) \cdot E \cdot \boldsymbol{n}|} .
$$

For simplicity, we assume that the coefficients of rolling and slipping friction are the same. Substituting the normal force from (2.12), we obtain

$$
\boldsymbol{F}_{c}=\mu a^{2} \frac{s_{c}\left(1-A^{*}\right)}{\beta_{3}}(\boldsymbol{n} \cdot E \cdot \boldsymbol{n})\left[\boldsymbol{n}-\nu \frac{(I-\boldsymbol{n} \boldsymbol{n}) \cdot E \cdot \boldsymbol{n}}{|(I-\boldsymbol{n n}) \cdot E \cdot \boldsymbol{n}|}\right] .
$$

We can deduce the relative velocity of slipping contact:

$$
\boldsymbol{V}^{s}=a s_{c}\left[\boldsymbol{\Omega} \times \boldsymbol{n}+\left\{1-B^{*}+\frac{\nu \beta_{6}(\boldsymbol{n} \cdot E \cdot \boldsymbol{n})}{|(I-\boldsymbol{n} \boldsymbol{n}) \cdot E \cdot \boldsymbol{n}|}\right\}(I-\boldsymbol{n n}) \cdot E \cdot \boldsymbol{n}\right] .
$$

We can also (as for rolling) compute the contact stresslet, neglecting terms of $O(\zeta)$ :

$$
S_{s}^{c}=+\mu a^{3} \frac{s_{c}\left(1-A^{*}\right) \beta_{5} \nu}{4 \beta_{3}}(\boldsymbol{n} \cdot E \cdot \boldsymbol{n})\left\{\frac{(I-\boldsymbol{n} \boldsymbol{n}) \cdot E \cdot \boldsymbol{n n}+\boldsymbol{n}(I-\boldsymbol{n n}) \cdot E \cdot \boldsymbol{n}}{|(I-\boldsymbol{n n}) \cdot E \cdot \boldsymbol{n}|}\right\} .
$$

The form of the friction model is such that the physical boundary between rolling and slipping motion is given by the position at which the relative velocity of the two spheres would be the same in rolling and slipping:

$$
\left(1-B^{*}-\beta_{4}\right)|(I-\boldsymbol{n n}) \cdot E \cdot \boldsymbol{n}|=-\nu \beta_{6}(\boldsymbol{n} \cdot E \cdot \boldsymbol{n}) .
$$




\subsection{Pair distribution function}

The pair distribution function $p(\boldsymbol{r})$ is defined as the probability of finding a particle centred at position $\boldsymbol{r}$ given that the test particle (particle 1) is centred at the origin. Because this function depends on the flow history, little can be ascertained about it without specifying the flow field.

In general, for each specific flow there will be five distinct regions of space in which to determine the probability distribution. These are:

(i) the bulk of space, for which the particle trajectories are unaffected by microscopic particle roughness and the probability distribution is the same as for the same flow containing smooth spheres;

(ii) the empty wake behind the particle of interest;

(iii) that part of the surface $s=s_{c}$ on which two particles are in rolling contact;

(iv) that part of the surface $s=s_{c}$ on which two particles are in sliding contact; and

(v) a surface in space separating region (i) from the empty wake (ii), if such a wake exists.

In each of these regions, the probability distribution is governed by the Liouville equation (Batchelor \& Green 1972a) (which is the high-Péclet-number form of the Smoluchowski equation):

$$
\boldsymbol{\nabla} \cdot[p(\boldsymbol{r}) \boldsymbol{V}(\boldsymbol{r})]=0 .
$$

The pair distribution function may be known for part or all of the bulk region. It was shown by Batchelor \& Green $(1972 a)$ that, for any material point which has come from infinity during the history of the flow, and has not been involved in a contact, the probability density at that point may be expressed as

$$
p(\boldsymbol{r})=q(s)
$$

in which $s=r / a$,

$$
\begin{aligned}
1 / q(s) & =(1-A(s)) \phi^{3}(s), \\
\phi(s) & =\exp \left[\int_{s}^{\infty} \frac{A\left(s^{\prime}\right)-B\left(s^{\prime}\right)}{1-A\left(s^{\prime}\right)} \frac{\mathrm{d} s^{\prime}}{s^{\prime}}\right],
\end{aligned}
$$

and $q(s) \rightarrow 1$ as $s \rightarrow \infty$. To find the probability density in any other region requires us first to specify the imposed flow field.

\section{Axisymmetric straining flow}

Our first flow field is a straining motion, $\boldsymbol{U}^{\infty}=E \cdot \boldsymbol{r}$, and we specify

$$
E=\left(\begin{array}{ccc}
E & 0 & 0 \\
0 & E & 0 \\
0 & 0 & -2 E
\end{array}\right) .
$$

The case $E>0$ is an axisymmetric straining motion with fluid entering along the $z$ direction and leaving in the $(x, y)$-plane. In the case $E<0$, the fluid enters in the $(x, y)$-plane and leaves in the $z$-direction. We define $\theta$ to be the angle subtended with the $z$-axis.

The trajectories swept out by the centre of particle 2 (relative to the centre of particle 1) are shown schematically in figure 1 . Note that, when the trajectory for smooth spheres reaches the boundary $s=2+\zeta$, it is deflected. Outside the limiting trajectory $\alpha \gamma$, along which the two particles just come into contact (at $\beta$ ), the behaviour of the system is exactly as it would be for perfectly smooth spheres. 


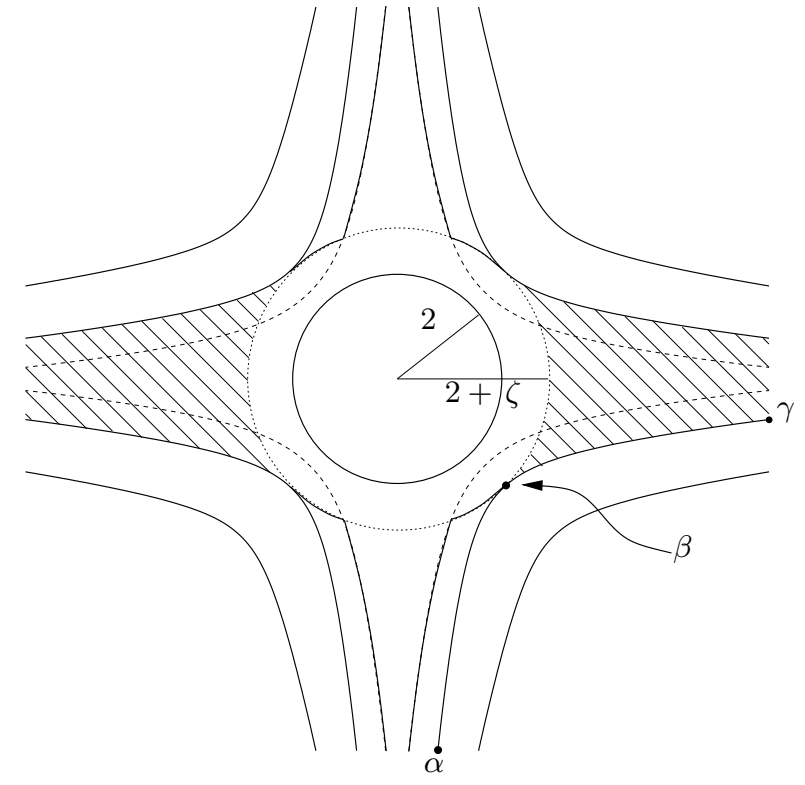

FIGURE 1. Trajectories of the centre of particle 2 relative to the centre of particle 1 in axisymmetric straining flow. When $E>0$ (biaxial expansion) the $z$-axis of symmetry is vertical in the diagram; when $E<0$ (uniaxial expansion or biaxial contraction) it is horizontal. In either case the pattern of trajectories is made three-dimensional by rotation about the $z$-axis. The dimensionless roughness height $\zeta$ is inflated for illustrative purposes.

The contact point $\beta$ subtends an angle $\theta_{0}$ with the $z$-axis; this angle is where $\boldsymbol{n} \cdot E \cdot \boldsymbol{n}=0$, i.e. $\theta_{0}=\arctan \left(2^{1 / 2}\right)$. Along trajectories which would, for smooth spheres, have the two spheres passing within a gap width less than $\zeta$ of each other, the particles come into contact and the model of Davis (1992) is used to determine the behaviour of the doublet of contacting particles. The particles remain in contact while they are in the compressive quadrant of the flow, and as they pass into the extensional quadrant of the flow they will separate, behaving as smooth spheres once the contact is over. It is important to note the "shadow" region in the wake of particle 1 (shaded in figure 1), which exists because the particle-particle contacts support compressive but not tensile forces; the pair density function $p$ will be zero in this region. On its border (trajectory $\beta \gamma$ ) there will be a high density of particles; this (two-dimensional) sheet region contains all the trajectories of particles which, in the smooth case, would have given trajectories in the (three-dimensional) shadow region.

\subsection{Calculation of the pair-distribution function}

The domain of interest can be divided up into three types of regions for calculation of the pair density $p$. First we have forbidden regions. These volumes have $p=0$, and are given by the 'excluded volume' region $s<2+\zeta$ and the shadow region (region (ii) of $\S 2.2$ ). Second, we have the volume (region (i) of $\S 2.2$ ) in which the probability is just as it would be if the spheres were perfectly smooth. This region is all of $s>2+\zeta$, except for the shadow region and its border. Finally, we have surface regions of high probability density, which result from particle-particle contact. In effect, these surfaces are where the excess probability density from the forbidden regions is to be found. On each such surface, we have a surface pair density function to describe the motion of the particles. The first surface (region (iii)) is part of the compressive quadrant of the boundary $s=2+\zeta$, on 
which the particles are in rolling contact and we have surface density $P^{r}$. In the rest of the compressive quadrant (region (iv)), the particles are in slipping contact, and we have surface probability density $P^{s}$. The third surface is the outer border of the shadow region (region $(\mathrm{v})$ ), given by rotating the curve $\beta \gamma$ and its reflection in the $(x, y)$-plane about the $z$-axis. On this sheet, we have a pair density $P^{s h}$. All three of these surface densities have dimensions of length (volume per area).

\subsubsection{Bulk region}

In the bulk, all the trajectories have come from infinity, and so (2.21) gives

$$
p(s)=q(s)
$$

for $s>2+\zeta$, except in the shadow region or on its border.

\subsubsection{Contact regions}

We note that there is a flux of particle pairs onto the compressive quadrant of the surface $s=s_{c} \equiv 2+\zeta$; the dimensionless flux is given by $q\left(s_{c}\right) V_{r}\left(s_{c}\right)$. The radial component of the relative velocity is

$$
V_{r}=a s(1-A)(\boldsymbol{n} \cdot E \cdot \boldsymbol{n})
$$

and thus, since the surface densities $P^{r}$ (and $P^{s}$ ) are defined by the Liouville balance equation, we have

$$
\boldsymbol{\nabla}_{s} \cdot\left[P^{r} \boldsymbol{V}^{r}\right]=-q(s) V_{r}=-a s_{c} \phi^{-3}\left(s_{c}\right) \boldsymbol{n} \cdot E \cdot \boldsymbol{n},
$$

where $\nabla_{s} \cdot \boldsymbol{u}$ is the surface divergence of $\boldsymbol{u}$, which may be expressed as

$$
\boldsymbol{\nabla}_{s} \cdot \boldsymbol{u}=\frac{1}{a s \sin \theta} \frac{\partial\left(\sin \theta u_{\theta}\right)}{\partial \theta}
$$

if $\boldsymbol{u}=u_{\theta} \boldsymbol{e}_{\theta}$. Substituting the form of $E$ into (2.17) and (2.13), we obtain the relative velocities of the two particles when in rolling and slipping contact, respectively:

$$
\begin{aligned}
& \boldsymbol{V}^{r}=3 a \beta_{4} s_{c} E \sin \theta \cos \theta \boldsymbol{e}_{\theta}, \\
& \boldsymbol{V}^{s}=a s_{c} E\left[3\left(1-B^{*}\right) \sin \theta \cos \theta \pm \nu \beta_{6}\left(1-3 \cos ^{2} \theta\right)\right] \boldsymbol{e}_{\theta},
\end{aligned}
$$

with the upper sign corresponding to the case $E>0$.

There is a critical value of $\theta$ at which slipping begins. In the case $E>0$ we have purely rolling motion for $\theta<\theta_{c}^{+}$, while for $\theta>\theta_{c}^{+}$there is some slipping. If $E<0$ the rolling occurs for $\theta>\theta_{c}^{-}$. The critical angle in each case is given by the point where the two velocities are identical (2.19):

$$
3\left(\beta_{4}+B^{*}-1\right) \sin \theta_{c}^{ \pm} \cos \theta_{c}^{ \pm}= \pm \nu \beta_{6}\left(1-3 \cos ^{2} \theta_{c}^{ \pm}\right),
$$

within the limits

$$
0<\theta_{c}^{+}<\theta_{0}<\theta_{c}^{-}<\pi / 2
$$

In the rolling region, we solve (3.4) with velocity (3.6) to obtain

$$
P^{r}=\frac{a s_{c}}{3 \beta_{4} \phi^{3}\left(s_{c}\right)},
$$

in which we have neglected the general solution $P^{r}=\sin ^{-2} \theta \cos ^{-1} \theta$ because it generates an unphysical surface source at $\theta=0$ (for $E>0$ ) and $\theta=\pi / 2$ (for $E<0$ ). In the slipping region we solve (3.4) with velocity (3.7) for the probability

$$
P^{s}=\frac{a s_{c} \sin \theta \cos \theta \phi^{-3}\left(s_{c}\right)}{\left[3\left(1-B^{*}\right) \sin \theta \cos \theta \pm \nu \beta_{6}\left(1-3 \cos ^{2} \theta\right)\right]} .
$$


In this case, the coefficient of the complementary solution is shown to be zero by matching $P^{r}$ and $P^{s}$ at $\theta=\theta_{c}$.

\subsubsection{Sheet region}

The surface bordering the shadow region is given, in polar coordinates, as

$$
s^{3} \sin ^{2} \theta \cos \theta=C_{c r} \phi^{3}(s), \quad C_{c r}=2(27)^{-1 / 2} s_{c}^{3} \phi^{-3}\left(s_{c}\right) .
$$

In this region, we can once more use the Liouville equation:

$$
\boldsymbol{\nabla}_{s} \cdot\left[P^{s h} \boldsymbol{V}\right]=0,
$$

with the gradient taking place along the sheet surface, and, because the particles are no longer in contact, the relative velocity of the two particles is

$$
\boldsymbol{V}=a s[(1-B(s)) \boldsymbol{E} \cdot \boldsymbol{n}+(B(s)-A(s))(\boldsymbol{n} \cdot \boldsymbol{E} \cdot \boldsymbol{n}) \boldsymbol{n}] .
$$

The upstream boundary condition is

$$
P_{0}^{s h}=P_{\theta_{0}}^{s}=\frac{a s_{c}}{3 \phi^{3}\left(s_{c}\right)\left(1-B^{*}\right)} .
$$

Equation (3.13) may be thought of as governing the flow of a fluid whose density at each point is given by $P^{s h}$. Then (3.13) is a condition of mass conservation of this fluid, and equivalently we may state that the flux of this fluid through each $s$-station in unit time is independent of $s$. Integrating between two arbitrary values of $s$ and applying the divergence theorem, it may be shown that $P^{s h}|\boldsymbol{V}| s \sin \theta$ is constant. Applying the upstream boundary condition, and noting that $1-B^{*}>0$, we may write

$$
P^{s h}|\boldsymbol{V}| s \sin \theta=\frac{2 a^{2} s_{c}^{3}}{(27)^{1 / 2} \phi^{3}\left(s_{c}\right)}|E| .
$$

\subsection{Form of the extra stress}

We seek to evaluate the integrals in (2.5). As discussed in $\S 2$, the term in (2.3) involving $e\left(\boldsymbol{x}_{0}, \boldsymbol{x}_{0}+\boldsymbol{r}\right)$ may be neglected provided that the angular integrations are carried out before the radial integration. We note that the axial symmetry of the flow and various regions implies that $\Sigma_{11}^{(p)}=\Sigma_{22}^{(p)}$ and $\Sigma_{i j}^{(p)}=0$ if $i \neq j$. Since the pressure of the entire system is arbitrary because of the incompressibility condition, and does not affect the flow of the suspension, we neglect isotropic terms and consider only the deviatoric part of the extra stress. The condition $\operatorname{tr}(\boldsymbol{\Sigma})=0$ then requires $\Sigma_{11}=-\frac{1}{2} \Sigma_{33}$, and so the deviatoric stress is a scalar multiple of the global rate of strain:

$$
\Sigma_{i j}=2 \mu^{*} E_{i j},
$$

where $\mu^{*}$ is the effective viscosity of the suspension. We can sum the contributions from all our regions, to express the result as

$$
\mu^{*}=\mu\left(1+\frac{5}{2} c+c^{2}\left[\frac{5}{2}+k_{\text {bulk }}+k_{\text {roll }}+k_{\text {slip }}+k_{\text {sheet }}\right]+O\left(c^{3}\right)\right),
$$

which is sufficient to specify all of the deviatoric stress components.

\subsubsection{Bulk region}

We start with the contribution from the bulk, in which $S^{c}=0$ and $p(\boldsymbol{r})=q(s)$ :

$$
\begin{aligned}
k_{b u l k}=-\frac{15}{8 \pi a^{3}} \int_{\text {bulk }} & \left(-2 K(s)+\left[-4 \cos ^{2} \theta\right] L(s)\right. \\
& \left.+\left(1-3 \cos ^{2} \theta\right)\left[\cos ^{2} \theta M(s)-\left(\frac{2}{3} L(s)+\frac{1}{3} M(s)\right)\right]\right) q(s) \mathrm{d} \boldsymbol{r} .
\end{aligned}
$$


Carrying out the angle integrals yields

$$
k_{\text {bulk }}^{ \pm}=\frac{15}{2} \int_{s_{c}}^{\infty} J_{ \pm} \mp v_{ \pm}\left[K+\frac{1}{3}\left(v_{ \pm}^{2}+1\right) L+\frac{1}{6}\left(\frac{9}{5} v_{ \pm}^{4}-2 v_{ \pm}^{2}+1\right) M\right] q(s) s^{2} \mathrm{~d} s,
$$

in which the upper sign corresponds to $E>0$,

$$
J_{+}(s)=K(s)+\frac{2}{3} L(s)+\frac{2}{15} M(s), \quad J_{-}(s)=0,
$$

and

$$
v_{ \pm}\left(1-v_{ \pm}^{2}\right)=C_{c r} \phi^{3}(s) / s^{3}, \quad 0<v_{+}<3^{-1 / 2}<v_{-}<1
$$

\subsubsection{Rolling surface}

The next contribution comes from that part of the compressive contact region in which the spheres are in rolling contact. Using (2.3) and (2.14),

$$
\begin{aligned}
\boldsymbol{\Sigma}_{\text {roll }}^{(p)}=\frac{15 c^{2} \mu}{4 \pi a^{3}} \int_{\text {roll }} P^{r}[K(s) E+[(E \cdot \boldsymbol{n}) \boldsymbol{n}+\boldsymbol{n}(E \cdot \boldsymbol{n})] L(s) \\
\left.\quad+(\boldsymbol{n} \cdot E \cdot \boldsymbol{n})\left[\left(\boldsymbol{n n}-\frac{1}{3} I\right) M(s)-\frac{2}{3} L(s) I\right]\right] \mathrm{d} S \\
\quad+\frac{9 c^{2} \mu}{16 \pi^{2} a^{3}} \frac{1}{4}\left(\beta_{2} \beta_{5} / \beta_{1}\right) \int_{\text {roll }}[((I-\boldsymbol{n n}) \cdot E \cdot \boldsymbol{n} \boldsymbol{n}+\boldsymbol{n}(I-\boldsymbol{n n}) \cdot E \cdot \boldsymbol{n})] P^{r} \mathrm{~d} S .
\end{aligned}
$$

This region is given by $0<\theta<\theta_{c}^{+}$and $\pi-\theta_{c}^{+}<\theta<\pi$ for $E>0$, which gives double the contribution from the $0<\theta<\theta_{c}^{+}$region, and $\theta_{c}^{-}<\theta<\pi-\theta_{c}^{-}$for $E<0$, which gives double the contribution from the region $\theta_{c}^{-}<\theta<\pi / 2$, and the volume integral was converted to a surface integral by posing

$$
P^{r}=p \mathrm{~d} r=a p \mathrm{~d} s
$$

in the immediate vicinity of the rolling surface. Thus $\mathrm{d} S=a^{2} s^{2} \sin \theta \mathrm{d} \theta \mathrm{d} \phi$.

Now we substitute our calculated probability, (3.10), to obtain

$$
\begin{aligned}
& k_{\text {roll }}^{ \pm}=\frac{3 \beta_{2} \beta_{5} s_{c}^{3}}{2560 \pi \beta_{1} \beta_{4} \phi^{3}\left(s_{c}\right)}\left[30 C_{1}^{ \pm}+10 C_{3}^{ \pm}-3 C_{5}^{ \pm}\right] \\
& \quad+\frac{s_{c}^{3}}{192 \beta_{4} \phi^{3}\left(s_{c}\right)}\left[10 C_{1}^{ \pm}\left(48 K^{*}+28 L^{*}+5 M^{*}\right)+5 C_{3}^{ \pm}\left(8 L^{*}+M^{*}\right)+9 C_{5}^{ \pm} M^{*}\right]
\end{aligned}
$$

in which we have denoted $X^{*} \equiv X\left(s_{c}\right)$ for $X=K, L$ or $M$, and

$$
C_{n}^{+}=1-\cos n \theta_{c}^{+}, \quad C_{n}^{-}=\cos n \theta_{c}^{-} .
$$

\subsubsection{Slipping surface}

The next contribution we consider comes from the rest of the contact surface, i.e. the region of slipping, which is equivalent to twice the region $\theta_{c}^{+}<\theta<\theta_{0}=\arctan \left(2^{1 / 2}\right)$ if $E>0$ and twice $\theta_{0}<\theta<\theta_{c}^{-}$if $E<0$. Using (2.18) and (3.11), the analysis proceeds as in the rolling case to obtain

$$
\begin{aligned}
k_{\text {slip }}=+\frac{9 s_{c}^{4}}{32 \pi \phi^{3}\left(s_{c}\right)} & \frac{\nu\left(1-A^{*}\right) \beta_{5}}{\beta_{3}}\left[I_{2}^{ \pm}-3 I_{4}^{ \pm}\right] \\
& \quad+\frac{5 s_{c}^{3}}{4 \phi^{3}\left(s_{c}\right)}\left[\left(6 K^{*}+2 L^{*}+M^{*}\right) I_{1}^{ \pm}+6\left(L^{*}-M^{*}\right) I_{3}^{ \pm}+9 M^{*} I_{5}^{ \pm}\right],
\end{aligned}
$$


where $X^{*}=X\left(s_{c}\right)$, as before, and

$$
I_{n}^{ \pm}= \begin{cases} \pm \int_{\theta_{c}^{ \pm}}^{\theta_{0}} \frac{\sin ^{2} \theta \cos ^{n} \theta \mathrm{d} \theta}{\left[3\left(1-B^{*}\right) \sin \theta \cos \theta \pm \nu \beta_{6}\left(1-3 \cos ^{2} \theta\right)\right]} & \text { for } n \text { odd } \\ \int_{\theta_{c}^{ \pm}}^{\theta_{0}^{ \pm}} \frac{\sin ^{3} \theta \cos ^{n} \theta \mathrm{d} \theta}{\left[3\left(1-B^{*}\right) \sin \theta \cos \theta \pm \nu \beta_{6}\left(1-3 \cos ^{2} \theta\right)\right]} & \text { for } n \text { even }\end{cases}
$$

\subsubsection{Sheet region}

The final contribution to our integral comes from the sheet which separates the bulk and shadow regions:

$$
\boldsymbol{\Sigma}_{\text {sheet }}^{(p)}=\frac{15 c^{2} \mu}{4 \pi a^{3}} \int_{\text {sheet }}\left(\frac{S\left(\boldsymbol{x}_{0}, \boldsymbol{x}_{0}+\boldsymbol{r}\right)}{(20 / 3) \pi \mu a^{3}}-E\right) P^{s h} \mathrm{~d} S,
$$

with $S$ defined in (2.4), the conversion to a surface integral being

$$
\mathrm{d} S=a^{2}|\boldsymbol{V}| \frac{\sin \theta}{V_{s}} s \mathrm{~d} s \mathrm{~d} \phi, \quad P^{s h}=p(\boldsymbol{r}) a \mathrm{~d} \theta
$$

and the integral being carried out along the sheet surface

$$
s^{3} \sin ^{2} \theta \cos \theta=C_{c r} \phi^{3}(s) \equiv 2 s_{c}^{3} \phi^{3}(s) \phi^{-3}\left(s_{c}\right) / 27^{1 / 2} .
$$

This surface is described by $\cos \theta=v_{ \pm}(s)$, with $v_{ \pm}(s)$ given by (3.22). We have

$$
\begin{aligned}
k_{\text {sheet }}=\frac{15}{4 \pi a^{3}} \int_{\text {sheet }} & \left(K(s)+2 \cos ^{2} \theta L(s)\right. \\
& \left.-\frac{1}{2}\left(1-3 \cos ^{2} \theta\right)\left[\cos ^{2} \theta M(s)-\left(\frac{2}{3} L(s)+\frac{1}{3} M(s)\right)\right]\right) P^{s h} \mathrm{~d} S .
\end{aligned}
$$

Now the probability is given by (3.16), and the relative velocity along the line of centres may be expressed as

$$
V_{s}=\mathrm{d} r / \mathrm{d} t=a s[(1-A(s)) \boldsymbol{n} \cdot E \cdot \boldsymbol{n}]=a E s(1-A(s))\left(1-3 \cos ^{2} \theta\right) .
$$

Thus,

$$
k_{\text {sheet }}^{ \pm}=\frac{5 s_{c}^{3}}{3^{1 / 2} \phi^{3}\left(s_{c}\right)} \int_{s_{c}}^{\infty}\left[K+\frac{1}{3}\left(3 v_{ \pm}^{2}+1\right) L+\frac{1}{6}\left(3 v_{ \pm}^{2}-1\right)^{2} M\right] \frac{\mathrm{d} s}{s(1-A)\left|1-3 v_{ \pm}^{2}\right|} .
$$

\subsection{Summary of viscosity results}

Throughout this section, the upper sign corresponds to $E>0$ and the lower to $E<0$. The viscosity is given by

$$
\mu^{ \pm}=\mu\left[1+\frac{5}{2} c+k_{ \pm} c^{2}+O\left(c^{3}\right)\right]
$$

in which

$$
k_{ \pm}=\frac{5}{2}+k_{\text {bulk }}^{ \pm}+k_{\text {roll }}^{ \pm}+k_{\text {slip }}^{ \pm}+k_{\text {sheet }}^{ \pm}
$$

and the individual terms are given by (3.20), (3.24), (3.26) and (3.33).

\subsubsection{Comparison with drops}

In order to check our work as far as possible, we compare our results with a similar calculation for spherical drops which come into contact due to their interfacial mobility but are not allowed to coalesce (Zinchenko 1984, equation (2.12)). If we put $\nu=0$, then there is no tangential friction between the particles and their interaction should be 
(a)

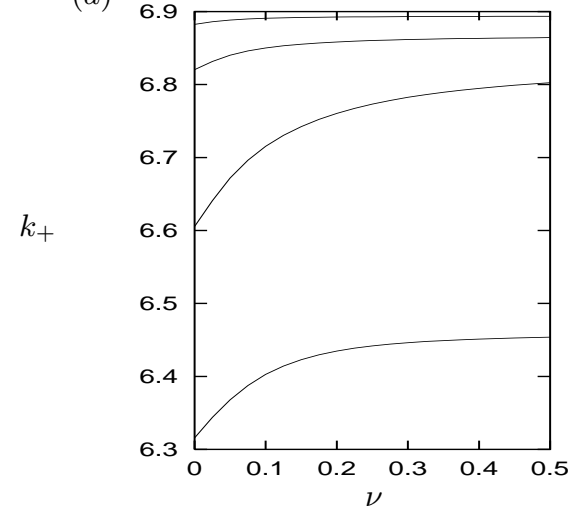

$(b)$

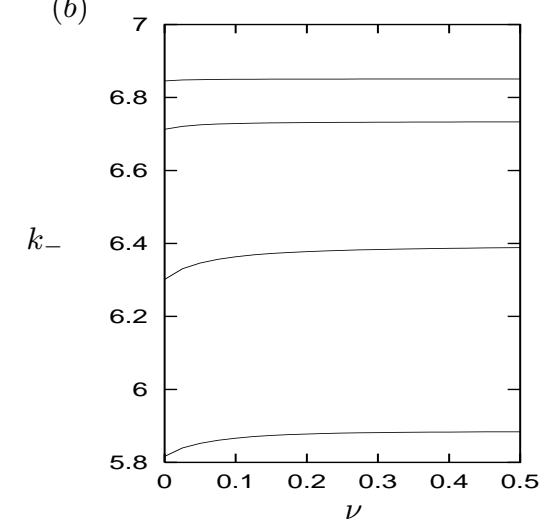

FiguRE 2. Plots of the $c^{2}$ viscosity coefficients $(a) k_{+}$and $(b) k_{-}$against the friction coefficient $\nu$ for $\zeta=10^{-7}, 10^{-5}, 10^{-3}$ and $10^{-2}$ (top to bottom). Figure modified from original paper.

similar to that between two drops. The information that the spheres are fluid or solid is contained in the form of the mobilities, and so if we set $s_{c}=2$ without using any other information, we should have the correct form for two fluid drops.

The critical angles become $\theta_{c}^{+}=0, \theta_{c}^{-}=\pi / 2$. The $C_{n}^{ \pm}$terms used in our expressions all become zero, and, if we denote $\left(I_{j}^{ \pm}\right)^{\prime}=\left(3^{1 / 2}\right) I_{j}^{ \pm} /\left(1-B^{*}\right)$, then

$$
\begin{array}{lll}
\left(I_{1}^{+}\right)^{\prime}=\left(3^{1 / 2}-1\right) / 3, & & \left(I_{1}^{-}\right)^{\prime}=1 / 3, \\
\left(I_{2}^{+}\right)^{\prime}=2\left(2^{1 / 2}\right) / 27, & & \left(I_{2}^{-}\right)^{\prime}=\left(2\left(2^{1 / 2}\right)-3\left(3^{1 / 2}\right)\right) / 27, \\
\left(I_{3}^{+}\right)^{\prime}=\left(3\left(3^{1 / 2}\right)-1\right) / 27, & & \left(I_{3}^{-}\right)^{\prime}=1 / 27, \\
\left(I_{4}^{+}\right)^{\prime}=2\left(2^{1 / 2}\right) / 45, & & \left(I_{4}^{-}\right)^{\prime}=2\left(2^{1 / 2}-3^{1 / 2}\right) / 45, \\
\left(I_{5}^{+}\right)^{\prime}=\left(9\left(3^{1 / 2}\right)-1\right) / 135, & & \left(I_{5}^{-}\right)^{\prime}=1 / 135 .
\end{array}
$$

Our viscosity result becomes

$$
\begin{aligned}
k_{ \pm}= & \frac{5}{2}+\frac{20}{\phi^{3}(2)\left(1-B^{*}\right)}\left[J_{ \pm}^{*} \mp \frac{1}{3^{1 / 2}}\left(K^{*}+\frac{4}{9} L^{*}+\frac{4}{45} M^{*}\right)\right] \\
& +\frac{40}{3^{1 / 2} \phi^{3}(2)} \int_{2}^{\infty}\left[K+\left(v_{ \pm}^{2}+\frac{1}{3}\right) L+\frac{1}{6}\left(3 v_{ \pm}^{2}-1\right)^{2} M\right] \frac{\mathrm{d} s}{s(1-A)\left|1-3 v_{+}^{2}\right|} \\
& +\frac{15}{2} \int_{2}^{\infty}\left\{J_{ \pm} \mp v_{ \pm}\left[K+\frac{1}{3}\left(v_{ \pm}^{2}+1\right) L+\frac{1}{6}\left(\frac{9}{5} v_{ \pm}^{4}-2 v_{ \pm}^{2}+1\right) M\right]\right\} q(s) s^{2} \mathrm{~d} s
\end{aligned}
$$

which agrees with Zinchenko's work when we note that

- Zinchenko's + corresponds to our -, and vice versa

- For solid spheres, Zinchenko's $\alpha=1$.

Both (3.37) and Zinchenko's (2.12) may be further simplified for the case of fully smooth, solid spheres by noting that $\phi(s) \rightarrow \infty$ as $s \rightarrow 2$ for solid spheres, and $v_{+}=0, v_{-}=1$, yielding equation (5.6) of Batchelor \& Green (1972a),

$$
k_{ \pm}=\frac{5}{2}+\frac{15}{2} \int_{2}^{\infty} J(s) q(s) s^{2} \mathrm{~d} s .
$$

\subsubsection{Numerical results and discussion}

An example set of results is shown in figure 2 , with $k_{ \pm}$plotted against $\nu$ for four sample values of $\zeta$. As expected, the limit $\zeta \rightarrow 0$ is that of smooth spheres, for which $k_{+}=k_{-}=k^{\text {smooth }} \approx 6.9$, independent of $\nu$. Batchelor \& Green (1972a), Zinchenko 
(1984) and Kim \& Mifflin (1985) reported $k^{\text {smooth }}=7.6,7.0$ and 7.1, respectively, with the small differences due to the accuracies of the mobility functions employed. The latter two are thought to be the most accurate, and our result is 6.9 , using a combination of the mobility data from Kim \& Mifflin (1985) and far- and near-field asymptotics. Unsurprisingly, the viscosities increase with increasing friction coefficient, $\nu$. The viscosity is always lower for rough spheres than for smooth ones, with the effect being more marked for larger roughness heights and for lower coefficients of friction.

In figure 3, we plot the individual contributions to the overall viscosity coefficient. Figure 3(a) shows the reduction caused by the excluded region $2<s<s_{c}$, expressed as a negative term,

$$
k_{e x c}=-\frac{15}{2} \int_{2}^{s_{c}} J(s) q(s) s^{2} \mathrm{~d} s=\frac{15}{2} \int_{s_{c}}^{\infty} J(s) q(s) s^{2} \mathrm{~d} s-k_{\text {smooth }},
$$

representing the isotropic part of the contribution from the bulk region for rough spheres minus that for smooth spheres. This reduction is relatively large in magnitude, reaching -1.1 at $\zeta=10^{-2}$. Because both the excluded volume region and the smooth-sphere probability distribution are spherically symmetric and do not depend on the sign of $E$, $k_{\text {exc }}$ is independent of the sign of $E$. The contribution shown in figure $3(b)$ from the sheet and wake regions combined contains the remainder of the contribution from the bulk (a negative contribution from the empty wake, $\left.k_{\text {wake }}=k_{\text {bulk }}-\left(k_{\text {smooth }}+k_{\text {exc }}\right)\right)$ added to the sheet region, which gives a positive contribution. The combination of the two terms is always negative.

Figures $3(c, d)$ show the contribution to the dissipation from the rolling and slipping contact regions, respectively. Because they are derived from the particle contacts, the dissipation values depend on $\nu$ as well as $\zeta$. The dissipation due to rolling increases as $\nu$ increases, primarily because the area of the contact surface in which rolling occurs increases. Similarly, the dissipation due to the slipping region decreases with increasing $\nu$. Although these two contributions are roughly the same order of magnitude, as the friction coefficient increases, the increase in dissipation in the rolling region dominates over the decrease in the slipping region. This is to be expected: addition of a source of dissipation (friction) to the problem increases the overall dissipation and hence the viscosity.

The lowest viscosity (i.e., the greatest deviation from the smooth-sphere viscosity) for a specific roughness height is at $\nu=0$, the limit in which all contact motion is slipping. In figure 2 we observe that, as $\nu$ increases, the curves asymptote to a value lower than the smooth limit. Thus, in figure 4 we plot the limits of $k_{ \pm}$for $\nu=0$ and $\nu \rightarrow \infty$ over a broad range of $\zeta$.

We observe that, in all four cases $(\nu \rightarrow 0$ and $\nu \rightarrow \infty, E>0$ and $E<0)$ the results are qualitatively similar, yielding a viscosity considerably lower than that for smooth spheres. In all cases, the viscosity for $\nu \rightarrow \infty$ is higher than that for $\nu \rightarrow 0$ and the viscosity for $E<0$ is lower than that for $E>0$. For very small roughness heights we can see that, as expected, all four viscosity coefficients converge to the same value $k_{\text {smooth }} \approx 6.9$; however, the difference is significant even for $\zeta=10^{-6}$ (for which a particle of $0.1 \mathrm{~mm}$ radius is molecularly smooth), and so roughness is expected always to play a rôle.

There is another possible effect of surface asperities on the viscosity of the suspension. The change in the maximum radius of each sphere may cause extra dissipation in the $O(c)$ term, which is caused by the dissipation around each particle in isolation. By the minimum dissipation theorem, the dissipation caused by any rough particle with maximum diameter $2 a(1+\zeta)$ is bounded above by the dissipation caused by a smooth sphere with exactly that diameter. We therefore assume this value (the sphere has effective ra- 
(a)
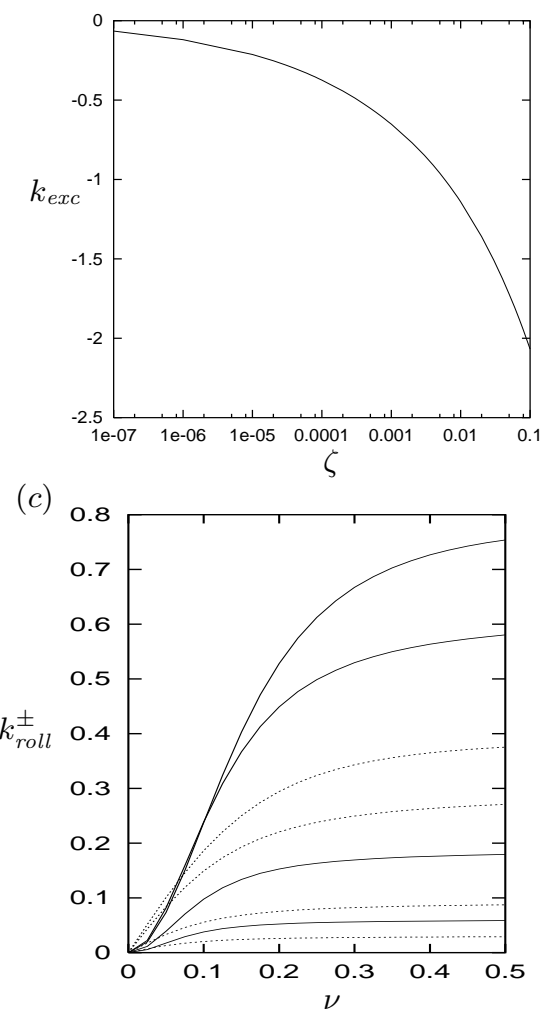

(b)
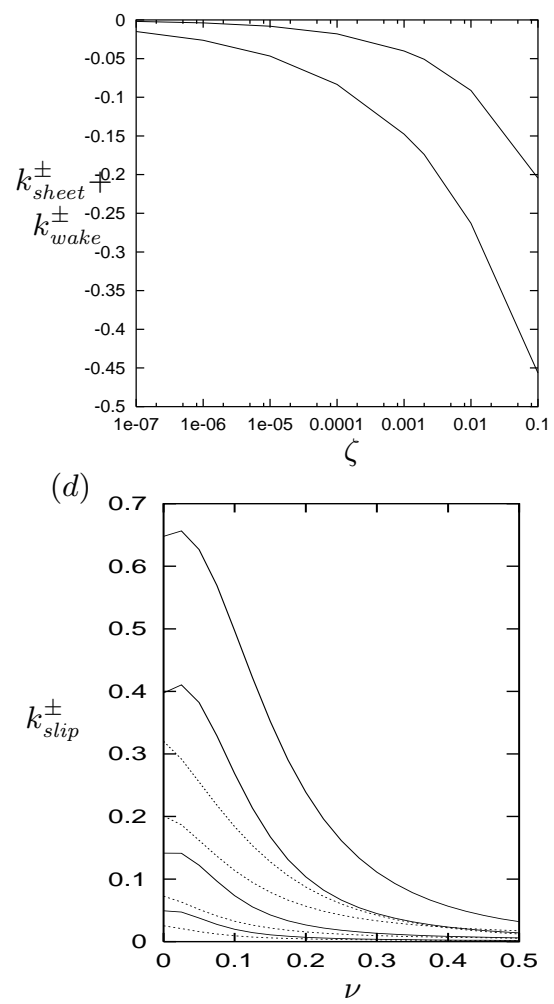

Figure 3 . The contributions to the $c^{2}$ viscosity coefficient $k_{ \pm}$from the $(a)$ excluded volume, $(b)$ sheet and wake, $(c)$ rolling contact and $(d)$ slipping contact regions of the straining flow. Since the contributions from the excluded region $s<s_{c}$, the sheet and the wake are independent of $\nu$, parts $(a)$ and $(b)$ are plotted against $\zeta$. In part $(b)$ the upper curve is for $E>0$ and the lower for $E<0$. Parts $(c)$ and $(d)$ are plotted against $\nu$ for $\zeta=10^{-2}, 10^{-3}, 10^{-5}$ and $10^{-7}$ from top to bottom, and the contributions for $E<0$ are solid curves where those for $E>0$ are dotted. Parts $(c)$ and $(d)$ are modified from the original paper.

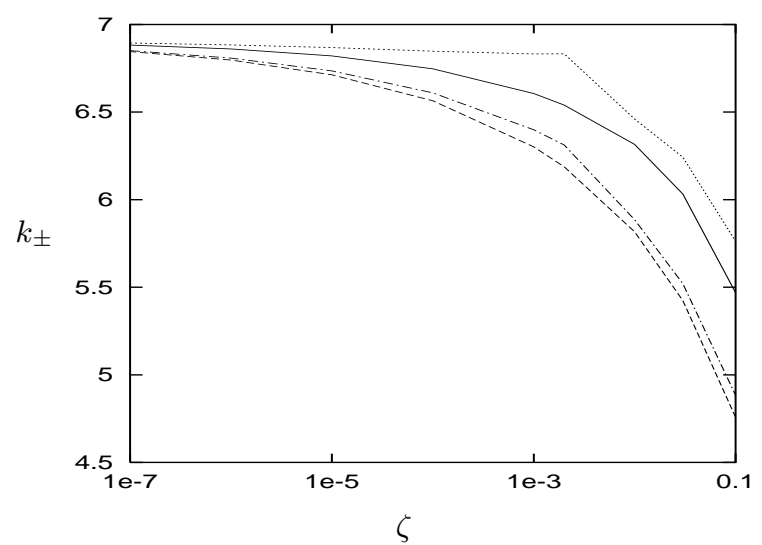

Figure 4 . Plot of the $c^{2}$ viscosity coefficient $k_{ \pm}$against the dimensionless roughness height $\zeta$ in the limits $\nu \rightarrow 0$ (no friction) and $\nu \rightarrow \infty$ (no slipping). The curves for $E>0$ (biaxial expansion) are solid for $\nu=0$ and short dashed for $\nu \rightarrow \infty$; the curves for $E<0$ (uniaxial expansion) are long dashed for $\nu=0$ and dot-dashed for $\nu \rightarrow \infty$. Figure modified from original paper. 
dius $\left.a_{\text {eff }}=a(1+\zeta)\right)$ as a worst case, in order to estimate the maximum effect of this dissipation. The effective volume concentration, which is proportional to $a_{\text {eff }}^{3}$, increases by a factor of $1+3 \zeta$ for $\zeta \ll 1$. Thus, our full adjusted viscosity becomes

$$
\mu^{ \pm}=\mu\left[1+\frac{5}{2} c(1+3 \zeta)+k_{ \pm} c^{2}+O\left(c^{3}\right)\right] .
$$

The two terms which change the viscosity from the smooth sphere case are

$$
\Delta \mu^{ \pm}=c \mu\left[\frac{15}{2} \zeta+\left(k_{ \pm}-k^{s m o o t h}\right) c\right]
$$

and so the $O\left(c^{2}\right)$ correction due to roughness is dominant for small roughness heights and/or large particle concentrations. For illustration, we choose a roughness height of $\zeta=2 \times 10^{-3}$ and a friction coefficient $\nu=0.25$, which are typical of values measured for glass and plastic spheres (Smart \& Leighton 1989; Smart et al. 1993; Zeng et al. 1996). In this case, $k_{+}-k^{\text {smooth }}=-0.15$ and $k_{-}-k^{\text {smooth }}=-0.7$, so that the $O\left(c^{2}\right)$ term is more important if $c>0.1$ for the biaxial expanding flow and $c>0.021$ for the biaxial contracting flow (bear in mind that the $O(c)$ term in (3.40) is an upper limit). These constraints both fall within the restriction of the analysis to dilute suspensions.

\section{Simple shear flow}

\subsection{Introduction}

For our second study, we consider simple shear flow, given in the absence of particles by $\boldsymbol{U}^{\infty}=(\dot{\gamma} y, 0,0) ;$ that is, $(2.1)$ with

$$
\begin{aligned}
& \boldsymbol{\Omega}=\frac{1}{2} \dot{\gamma}(0,0,-1), \\
& E=\frac{1}{2} \dot{\gamma}\left(\begin{array}{ccc}
0 & 1 & 0 \\
1 & 0 & 0 \\
0 & 0 & 0
\end{array}\right)
\end{aligned}
$$

We assume that $\dot{\gamma}>0$ throughout this section, with the symmetry of the problem providing the results for $\dot{\gamma}<0$ : if $\dot{\gamma} \rightarrow-\dot{\gamma}$ then $\Sigma_{12} \rightarrow-\Sigma_{12}$, and the other stress terms are unchanged, and so the viscosity and normal stresses are unchanged.

Let us consider simple shear flow containing two spheres, one of which is centred at the origin. The relative trajectories (Zinchenko 1984) are given by

$$
y^{2}=a^{2} \phi^{2}(s)\left[\xi_{2}+\Psi(s)\right], \quad z=a \phi(s) \xi_{3},
$$

in which

$$
\Psi(s)=\int_{s}^{\infty} \frac{B\left(s^{\prime}\right) s^{\prime} \mathrm{d} s^{\prime}}{\left(1-A\left(s^{\prime}\right)\right) \phi^{2}\left(s^{\prime}\right)}
$$

and $\phi(s)$ is as defined in (2.23). Along each individual trajectory, $\xi_{2}$ and $\xi_{3}$ are constant.

We use the Cartesian coordinates $(x, y, z)$ given above in parallel with spherical polar coordinates $(s, \theta, \phi)$ given by

$$
\begin{aligned}
& x=a s \cos \theta, \\
& y=a s \sin \theta \cos \phi, \\
& z=a s \sin \theta \sin \phi .
\end{aligned}
$$

Using these coordinates, we note the following equalities:

$$
\boldsymbol{n} \cdot \boldsymbol{E} \cdot \boldsymbol{n}=\dot{\gamma} \sin \theta \cos \theta \cos \phi,
$$



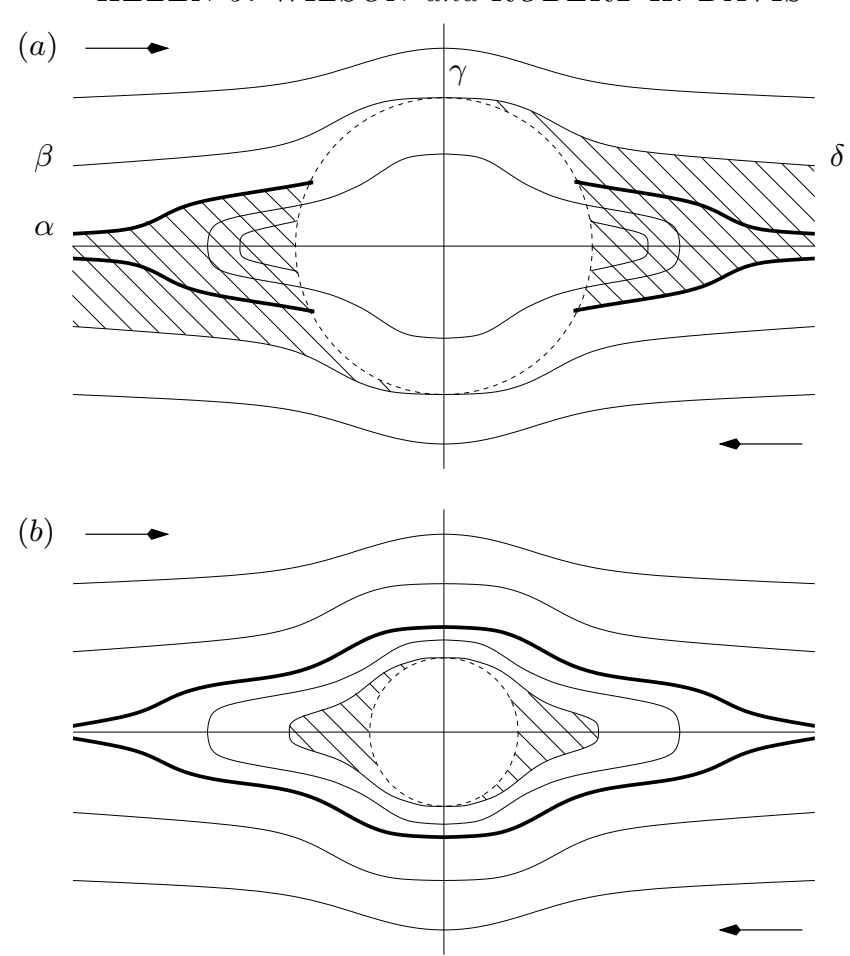

FIGURE 5. Trajectories in the $(x, y)$ plane of the centre of particle 2 relative to particle 1 in shear flow, $(a)$ when all closed orbits pass within the contact surface, and $(b)$ when some closed orbits are entirely outside the contact surface. The bold trajectory represents symbolically the trajectory dividing closed orbits from open trajectories. The dotted circles represent the contact surface $s=s_{c} \equiv 2+\zeta$. The shaded region represents forbidden areas where there will be no particles once the flow is well-established; and on the edge of this region there may be a high density of particles.

$$
(I-\boldsymbol{n n}) \cdot \boldsymbol{E} \cdot \boldsymbol{n}=\frac{1}{2} \dot{\gamma}\left(\begin{array}{c}
\sin \theta \cos \phi\left(1-2 \cos ^{2} \theta\right) \\
\cos \theta\left(1-2 \sin ^{2} \theta \cos ^{2} \phi\right) \\
-2 \sin ^{2} \theta \cos \theta \sin \phi \cos \phi
\end{array}\right) .
$$

In the absence of surface irregularities, there are two types of relative trajectories:

- Open trajectories which arrive from infinity and depart to infinity

- Closed trajectories or cycles. These form the symmetric region

$$
s^{2} \sin ^{2} \theta \cos ^{2} \phi<\phi^{2}(s) \Psi(s) .
$$

The pair density function is not known for the closed orbits, and so the $O\left(c^{2}\right)$ viscosity correction is indeterminate (Batchelor \& Green 1972a).

When the surfaces of the spheres exhibit surface irregularities, however, the pattern of relative trajectories is more complicated. Typical surface asperities have height around $10^{-3}-10^{-2}$ of a particle radius (Smart \& Leighton 1989). For two spheres interacting in the $(x, y)$-plane, this size is large enough that all the closed orbits in that plane will be affected by contact, and so their symmetry will be broken and the indeterminacy they cause is abolished. This scenario is illustrated in figure $5(a)$.

On the other hand, when the two particles are initially offset in the $z$-direction, the region of the contact surface which intersects with the sheet of trajectories of sphere 2 is much smaller (sometimes nonexistent), and there will be entire closed trajectories which 
pass outside it. This case is shown in figure $5(b)$. It is important to note that both of these streamline patterns can occur simultaneously in the same flow. Indeed, for any roughness height large enough to break some closed trajectories, there will be both patterns in the same flow, as there are closed orbits whose distance of closest approach is arbitrarily large. This means that the viscosity is always undetermined (as discussed in $§ 4.2$ ).

If the height of the surface asperities is very small (i.e. the particles are exceptionally smooth), it is possible for all of the contact surface to fall within the region of closed trajectories. This case occurs when the minimum distance of approach of the trajectory dividing closed orbits from open trajectories is greater than the roughness height. Mathematically, it requires that

$$
\phi^{2}\left(s_{c}\right) \Psi\left(s_{c}\right)>s_{c}^{2}
$$

and we have determined (using a numerical interpolation) that this inequality is satisfied when $\zeta<2.110 \times 10^{-4}$. This roughness height is much smaller than typically encountered, and so it is reasonable to expect that there will be some open trajectories which intersect the contact surface. In this case, as discussed by Rampall, Smart \& Leighton (1997), the probability distribution may be calculated in the plane of shear, and so the viscosity of a monolayer suspension of spheres may be calculated exactly. However, if the roughness height is smaller than the distance of closest approach $\left(\zeta<2.110 \times 10^{-4}\right)$, then the surface roughness has no effect, the analysis of Batchelor \& Green (1972a) is still valid, and the normal stress differences are zero.

\subsection{Calculation of the pair-distribution function}

In order to calculate the probability distribution $p$ in simple shear flow, we must consider the following regions of the flow:

- the bulk of the flow: trajectories which originate at infinity and either reach the contact surface or depart to infinity without ever intersecting the contact surface

- closed orbits which do not intersect the contact surface

- the shadow region in which no particles can be found because of contacts which exert compressive but not tensile forces; here $p(\boldsymbol{r})=0$

- the rolling region of the contact surface

- the slipping region of the contact surface

- the border of the shadow region.

The first two of these regions correspond to region (i) of $\S 2.2$; the probability density there is unaffected by particle friction.

Unfortunately, the probability distribution is not known on the closed orbits. This fact prevents us, as it prevented Batchelor \& Green (1972a) and Zinchenko (1984), from calculating exact viscosity values in this flow unless some distribution is assumed at a given instant in time. To demonstrate the problem of undetermined probabilities in the closed-orbit region, we have chosen two plausible distributions for smooth spheres. In the first case, we take the probability distribution to be $p(\boldsymbol{r})=q(s)$ everywhere, a distribution which is continuous at the edge of the region of closed orbits. As shown by Batchelor \& Green $(1972 a)$, the $c^{2}$ viscosity coefficient in this case is $k=2.5+7.5 \int J q s^{2} \mathrm{~d} s \approx 6.9$. In the second case, we assume that the region of closed orbits is well-stirred initially, so that $p=1$ inside this region. As time passes, the probability distribution within the region of closed orbits will fluctuate, but effectively it will oscillate about this value. Therefore it is reasonable to consider a distribution in the outer region which has settled down to its long-term value of $p=q$, while the closed-orbit region instantaneously has $p=1$. This distribution gives an instantaneous coefficient of $k \approx 5.9$. Since the difference between these two situations is comparable to the change in the $c^{2}$ viscosity coefficient 
due to roughness obtained for extensional flow, we cannot hope to deduce definitive information about the effect of surface roughness on the shear viscosity by performing further calculations. Indeed, these calculations would entail a considerable amount of work for conclusions which would be uncertain at the very best.

However, as we shall see, the normal stresses $N_{1}=\Sigma_{11}-\Sigma_{22}$ and $N_{2}=\Sigma_{22}-\Sigma_{33}$ are unaffected by the probability distribution on the closed orbits. We may calculate them without making any further assumptions.

\subsubsection{Bulk region}

In the first region described above, the fact that the particles are not smooth has no effect on the flow, and the particle trajectories are coming from infinity, and so (2.21) applies. The velocity in the first region is given by (2.7), and so the flux of pairs from this region onto the contact surface is given by

$$
q\left(s_{c}\right) V_{r}\left(s_{c}\right)=a q\left(s_{c}\right) s_{c}\left(1-A\left(s_{c}\right)\right)(\boldsymbol{n} \cdot \mathbf{E} \cdot \boldsymbol{n}) .
$$

In the region of closed orbits, however, the argument used by Batchelor \& Green (1972a) can only give us

$$
p(\boldsymbol{r})=C\left(\xi_{2}, \xi_{3}\right) q(s)
$$

where $\xi_{2}, \xi_{3}$ are the invariants of (4.3), and no information is available about the form of $C\left(\xi_{2}, \xi_{3}\right)$ unless other effects (such as Brownian motion or longer range forces) are included.

\subsubsection{Contact surface}

On the rolling portion of the contact surface, the relative velocity of the two spheres is given by (2.13), and that for slipping is given by (2.17). The boundary between rolling and slipping is the point at which the velocity is the same by either mechanism (2.19):

$$
\left(1-B^{*}-\beta_{4}\right)|(\boldsymbol{I}-\boldsymbol{n} \boldsymbol{n}) \cdot E \cdot \boldsymbol{n}|=-\nu \beta_{6}(\boldsymbol{n} \cdot E \cdot \boldsymbol{n}),
$$

which turns out, for realistic values of $\nu$ ( $\nu \leq 0.5$, say), to encompass a very small region of the contact surface. Most of the contact surface, therefore, is a slipping region. In particular, the boundary condition $P^{c}=0$ on the edge of the forbidden region must be applied to the edge of the slipping region.

Unfortunately, the form of $|(I-\boldsymbol{n n}) \cdot \boldsymbol{E} \cdot \boldsymbol{n}|$, when expressed in terms of the angles $\theta$ and $\phi$, is sufficiently complicated that the partial differential equation which results from the Liouville equation in this case cannot be solved analytically. In turn, the boundary conditions for the rolling region are not known analytically, so that region must also be investigated numerically.

Here, we give the pair of equations which must be solved numerically. To construct them, first we substitute $\boldsymbol{E}, \boldsymbol{\Omega}$ and $\boldsymbol{n}$ into the forms of $\boldsymbol{V}(2.13,2.17)$. We express all the quantities in trigonometric terms and use $X=\sin \phi$ and $Y=\sin \theta$, substituting into the Liouville equation

$$
\boldsymbol{\nabla} \cdot\left[P^{c} \boldsymbol{V}\right]=-a s \dot{\gamma} \phi^{-3}\left(s_{c}\right) \sin \theta \cos \theta \cos \phi
$$

to obtain

$$
\frac{\partial}{\partial X}\left(P^{c} X[1-\alpha(X, Y)]\right)-\frac{\partial}{\partial Y}\left(P^{c} Y\left[1-\left(1-2 Y^{2}\right) \alpha(X, Y)\right]\right)=-4 a \phi^{-3}\left(s_{c}\right) Y^{2},
$$

in which

$$
\alpha(X, Y)= \begin{cases}\beta_{4} & \text { for rolling } \\ 1-B^{*}-\nu \beta_{6} Y /|f(X, Y)| & \text { for slipping }\end{cases}
$$


and

$$
f^{2}(X, Y)=\frac{\left[\left(1-2 Y^{2}\right)^{2}+X^{2} Y^{2}\left(3-4 Y^{2}\right)\right]}{4\left(1-X^{2}\right)\left(1-Y^{2}\right)} .
$$

This equation is solved numerically in the region

$$
\{0 \leq X \leq 1 ; 0 \leq Y \leq 1\} \cap\left\{Y^{2}\left(1-X^{2}\right)>\phi^{2}\left(s_{c}\right) \Psi\left(s_{c}\right) / s_{c}^{2}\right\}
$$

using the method of characteristics, with boundary condition $P^{c}=0$ on the edge of the forbidden region, where $Y^{2}\left(1-X^{2}\right)=\phi^{2}\left(s_{c}\right) \Psi\left(s_{c}\right) / s_{c}^{2}$. The solution can be extrapolated to the rest of the contact surface using symmetry considerations.

\subsubsection{Border of the shadow region}

The border of the shadow region may consist of

- closed orbits, on which we cannot determine the probability density without ad hoc assumptions (figure $5 b$ ), and

- two surfaces in the wake of sphere 1 , of finite extent in the $y$ and $z$ directions, and semi-infinite in the $x$-direction (figure $5 a$ ).

We consider only the case when the latter two surfaces exist (though there will also be closed orbits), since the results when there are only closed orbits are exactly the same as for smooth spheres.

In a similar manner to that used in $\S 3$, we note that, since there is no flux of probability onto or off the sheet, the Liouville equation is equivalent to mass conservation on the sheet. We parametrise the sheet using the trajectory length, $l$, and the azimuthal angle at the point of detachment, $\tilde{\phi}$. We also define a quantity $\mathrm{d} h$ to be the length element on the sheet perpendicular to the velocity.

We consider a narrow band of trajectories leaving the sphere, and integrate the Liouville equation (3.4), with velocity (2.7) over the section of the surface they pass through between leaving the sphere and having travelled a dimensionless distance $l$. We apply the divergence theorem, noting that the contribution from edges parallel to $\boldsymbol{V}$ is zero, and using $\boldsymbol{n}=\boldsymbol{V} /|V|$ on the remaining edges and the upstream boundary conditions,

$$
P^{s h}|V| \mathrm{d} S=\frac{1}{2} a^{2} P_{0}^{s h} s_{c}^{3}\left(2-B^{*}\right) \dot{\gamma}|\cos \tilde{\phi} \mathrm{d} \tilde{\phi}| \mathrm{d} l .
$$

Now, since $\mathrm{d} l$ is the length element parallel to $\boldsymbol{V}$, we have $\mathrm{d} l / \mathrm{d} t=|V|$ and of course $a \mathrm{~d} s / \mathrm{d} t=V_{s}$. Now $\left|V_{s}\right|=|\boldsymbol{V} \cdot \boldsymbol{n}|=\dot{\gamma}(1-A)|x y| / a s$, so

$$
P^{s h} \mathrm{~d} S=\frac{P_{0}^{s h} a^{4} s_{c}^{3}\left(2-B^{*}\right) s|\cos \tilde{\phi} \mathrm{d} \tilde{\phi} \mathrm{d} s|}{2(1-A)|x y|} .
$$

Now the value $P_{0}^{s h}$ is equal to the value of $P^{c}$ (which is calculated numerically) at $\theta=\pi / 2$. Because the upstream boundary condition is only known numerically, this equation, like that in $\S 4.2 .2$, may only be used in numerical calculations. However, once the probability has been calculated on the contact surface, $P_{0}^{s h}$ is known and therefore $P^{s h} \mathrm{~d} S$ may be found without further integration.

We need information about the shape of the sheet in order to express $x$ and $y$ as a function of $s$ and $\tilde{\phi}$. We use the form of the trajectories (4.3), and, applying the initial condition $x=0, y=a s \cos \tilde{\phi}$ at $s=s_{c}$ to determine $\xi_{2}$ and $\xi_{3}$, we obtain

$$
\begin{aligned}
\frac{x^{2}}{a^{2} s^{2}} & =1-\frac{\phi^{2}(s)}{\phi^{2}\left(s_{c}\right)} \frac{s_{c}^{2}}{s^{2}}+\frac{\phi^{2}(s)}{s^{2}}\left[\Psi\left(s_{c}\right)-\Psi(s)\right], \\
\frac{y^{2}}{a^{2} s^{2}} & =\frac{\phi^{2}(s)}{\phi^{2}\left(s_{c}\right)} \frac{s_{c}^{2}}{s^{2}} \cos ^{2} \tilde{\phi}-\frac{\phi^{2}(s)}{s^{2}}\left[\Psi\left(s_{c}\right)-\Psi(s)\right] .
\end{aligned}
$$




\subsection{Calculation of the normal stresses}

Using (2.5-2.6) and (4.2) we can show that the normal stresses $N_{1}=\Sigma_{11}-\Sigma_{22}, N_{2}=$ $\Sigma_{22}-\Sigma_{33}$ will be given by

$$
\begin{aligned}
N_{1}= & \frac{15 c^{2} \mu \dot{\gamma}}{4 \pi} \int_{r \geq 2 a} n_{1} n_{2}\left(n_{1}^{2}-n_{2}^{2}\right) M(s) p(\boldsymbol{r}) \frac{\mathrm{d} \boldsymbol{r}}{a^{3}} \\
& +\frac{9 c^{2} \mu \dot{\gamma}}{32 \pi} \int_{\text {contact }} \beta_{5}\left[\frac{\left(\boldsymbol{F}_{c} \cdot \boldsymbol{n}\right)}{\mu \dot{\gamma} \pi a^{2}}\left(n_{1}^{2}-n_{2}^{2}\right)-\frac{F_{1}^{c} n_{1}-F_{2}^{c} n_{2}}{\mu \dot{\gamma} \pi a^{2}}\right] p(\boldsymbol{r}) \frac{\mathrm{d} \boldsymbol{r}}{a^{3}}+O\left(c^{3}\right), \\
N_{2}= & \frac{15 c^{2} \mu \dot{\gamma}}{4 \pi} \int_{r \geq 2 a} n_{1} n_{2}\left[L(s)+\left(n_{2}^{2}-n_{3}^{2}\right) M(s)\right] p(\boldsymbol{r}) \frac{\mathrm{d} \boldsymbol{r}}{a^{3}} \\
& +\frac{9 c^{2} \mu \dot{\gamma}}{32 \pi} \int_{\text {contact }} \beta_{5}\left[\frac{\left(\boldsymbol{F}_{c} \cdot \boldsymbol{n}\right)}{\mu \dot{\gamma} \pi a^{2}}\left(n_{2}^{2}-n_{3}^{2}\right)-\frac{\left(F_{2}^{c} n_{2}-F_{3}^{c} n_{3}\right)}{\mu \dot{\gamma} \pi a^{2}}\right] p(\boldsymbol{r}) \frac{\mathrm{d} \boldsymbol{r}}{a^{3}}+O\left(c^{3}\right),
\end{aligned}
$$

and the contribution to the integrals from $e$ is zero. We express the sub-terms of these expressions as

$$
N_{i}=\frac{15 c^{2} \mu|\dot{\gamma}|}{4 \pi}\left(\hat{N}_{\text {bulk }}^{i}+\hat{N}_{\text {contact }}^{i, S}+\hat{N}_{\text {sheet }}^{i}\right)+\frac{9 c^{2} \mu|\dot{\gamma}|}{32 \pi} \hat{N}_{\text {contact }}^{i, D}+O\left(c^{3}\right),
$$

where $i=1$ or 2 .

\subsubsection{Bulk region}

In the bulk, $\boldsymbol{F}=\mathbf{0}$ since the particles are not in contact, and the contributions to the normal stresses are thus

$$
\begin{gathered}
\hat{N}_{b u l k}^{1}=\int_{r \geq 2 a} n_{1} n_{2}\left(n_{1}^{2}-n_{2}^{2}\right) M(s) p(\boldsymbol{r}) \mathrm{d} \boldsymbol{s}, \\
\hat{N}_{b u l k}^{2}=\int_{r \geq 2 a} n_{1} n_{2}\left\{L(s)+\left(n_{2}^{2}-n_{3}^{2}\right) M(s)\right\} p(\boldsymbol{r}) \mathrm{d} \boldsymbol{s} .
\end{gathered}
$$

Throughout the bulk, we have

$$
p(\boldsymbol{r})=C\left(\xi_{2}, \xi_{3}\right) q(s)
$$

in which $C \equiv 1$ except on closed orbits. Now $s$ is an even function of $n_{1}$, whereas the integrand in each case is an odd function of $n_{1}$. Thus the contribution from any trajectory along which $n_{1}$ is symmetrically positive and negative must be zero. This case includes any closed orbits and also the unbounded trajectories which do not intersect the contact surface.

The only nonzero contribution is therefore from the region of trajectories entering from infinity and intersecting the contact surface. Because they are coming from infinity, these trajectories satisfy (2.21). This region can be expressed as

$$
\{x y<0\} \cap\left\{y^{2}+z^{2} \leq a^{2} \phi^{2}(s)\left[\frac{s_{c}^{2}}{\phi^{2}\left(s_{c}\right)}-\Psi\left(s_{c}\right)+\Psi(s)\right]\right\} \cap\left\{y^{2} \geq a^{2} \phi^{2}(s) \Psi(s)\right\},
$$

which becomes

$$
\begin{aligned}
&\{\sin \theta \cos \theta \cos \phi<0\} \cap\left\{\sin ^{2} \theta \leq \frac{\phi^{2}(s)}{s^{2}}\left[\frac{s_{c}^{2}}{\phi^{2}\left(s_{c}\right)}-\Psi\left(s_{c}\right)+\Psi(s)\right]\right\} \\
& \cap\left\{\sin ^{2} \theta \cos ^{2} \phi \geq \frac{\phi^{2}(s)}{s^{2}} \Psi(s)\right\} .
\end{aligned}
$$


This region only exists if $\Psi\left(s_{c}\right) \phi^{2}\left(s_{c}\right) \leq s_{c}^{2}$, i.e. if there is an intersection between the contact surface and the open trajectories. As discussed in $\S 4.1$, this case corresponds to the reasonable constraint $\zeta>2 \times 10^{-4}$.

Performing the integral over $\phi$ first and then over $\theta$, the first integral (4.26) becomes

$$
\begin{aligned}
\hat{N}_{b u l k}^{1}= & \frac{-4}{3 \phi^{3}\left(s_{c}\right)}\left(s_{c}^{2}-\phi^{2}\left(s_{c}\right) \Psi\left(s_{c}\right)\right)^{3 / 2} \times \\
& \int_{s_{c}}^{\infty} \frac{M(s)}{(1-A(s)) s^{3}}\left[s^{2}+\phi^{2}(s)\left(\frac{-s_{c}^{2}}{\phi^{2}\left(s_{c}\right)}-2 \Psi(s)+\Psi\left(s_{c}\right)\right)\right] \mathrm{d} s,
\end{aligned}
$$

and the second integral (4.27)

$$
\begin{aligned}
\hat{N}_{\text {bulk }}^{2}= & \frac{-4}{15 \phi^{3}\left(s_{c}\right)}\left(s_{c}^{2}-\phi^{2}\left(s_{c}\right) \Psi\left(s_{c}\right)\right)^{3 / 2} \times \\
& \int_{s_{c}}^{\infty}\left\{\frac{M(s) \phi^{2}(s)}{(1-A(s)) s^{3}}\left[\frac{s_{c}^{2}}{\phi^{2}\left(s_{c}\right)}+5 \Psi(s)-\Psi\left(s_{c}\right)\right]+\frac{5 L(s)}{s(1-A(s))}\right\} \mathrm{d} s .
\end{aligned}
$$

\subsubsection{Contact surface}

In the contact region we have calculated $P^{c}$ numerically, and so the total stress contributions $\hat{N}_{\text {contact }}^{i, S}$ and $\hat{N}_{\text {contact }}^{i, D}$ must also be calculated numerically. As for strain, we convert the volume integrals to surface integrals using $P^{c}=a p \mathrm{~d} s$. We substitute (4.8) and (4.9) into (2.14) and (2.18) for the force dipole. Substituting the definition $\mathrm{d} S=a^{2} s_{c}^{2} \sin \theta \mathrm{d} \theta \mathrm{d} \phi$ and using the variables we introduced for calculating the probability, $X=\sin \phi, Y=\sin \theta$, we obtain

$$
\begin{gathered}
\hat{N}_{\text {contact }}^{1, S}=-2 M^{*} s_{c}^{2} \int_{-1}^{1} \int_{0}^{1} Y^{2}\left(1-2 Y^{2}+Y^{2} X^{2}\right) \frac{P^{c}}{a} \mathrm{~d} Y \mathrm{~d} X, \\
\hat{N}_{\text {contact }}^{2, S}=-2 s_{c}^{2} \int_{-1}^{1} \int_{0}^{1} Y^{2}\left(L^{*}+Y^{2}\left(1-2 X^{2}\right) M^{*}\right) \frac{P^{c}}{a} \mathrm{~d} Y \mathrm{~d} X, \\
\hat{N}_{\text {roll }}^{1, D}=\frac{2 \beta_{2} \beta_{5}}{\pi \beta_{1}} s_{c}^{2} \int_{-1}^{1} \int_{0}^{1} Y^{2}\left(1-2 Y^{2}+Y^{2} X^{2}\right) \frac{P^{c}}{a} \mathrm{~d} Y \mathrm{~d} X, \\
\hat{N}_{\text {roll }}^{2, D}=\frac{\beta_{2} \beta_{5}}{\pi \beta_{1}} s_{c}^{2} \int_{-1}^{1} \int_{0}^{1} Y^{2}\left(2 Y^{2}\left(1-2 X^{2}\right)-1\right) \frac{P^{c}}{a} \mathrm{~d} Y \mathrm{~d} X, \\
\hat{N}_{\text {slip }}^{1, D}=+\frac{2\left(1-A^{*}\right) \beta_{5}}{\pi \beta_{3}} s_{c}^{3} \nu \int_{-1}^{1} \int_{0}^{1} \frac{Y^{3}}{f(X, Y)}\left(1-2 Y^{2}+Y^{2} X^{2}\right) \frac{P^{c}}{a} \mathrm{~d} Y \mathrm{~d} X, \\
\hat{N}_{\text {slip }}^{2, D}=+\frac{\left(1-A^{*}\right) \beta_{5}}{\pi \beta_{3}} s_{c}^{3} \nu \int_{-1}^{1} \int_{0}^{1} \frac{Y^{3}}{f(X, Y)}\left(2 Y^{2}\left(1-2 X^{2}\right)-1\right) \frac{P^{c}}{a} \mathrm{~d} Y \mathrm{~d} X,
\end{gathered}
$$

with $f(X, Y)$ as defined in (4.18), so that

$$
f^{2}(X, Y)=\frac{|(I-\boldsymbol{n n}) \cdot E \cdot \boldsymbol{n}|^{2}}{\dot{\gamma}^{2}\left(1-X^{2}\right)\left(1-Y^{2}\right)} .
$$

\subsubsection{Border of the shadow region}

On the sheet surface, the particles are not in contact, and so there is no contribution to the stress from the contact force dipole. Therefore (4.26) and (4.27) hold, as they did 
in the bulk. On the sheet (4.20) holds, and so we have

$$
\begin{aligned}
\int_{\text {sheet }}[\ldots] p(\boldsymbol{r}) \mathrm{d} \boldsymbol{r} & =\int_{\text {sheet }}[\ldots] P^{s h} \mathrm{~d} S \\
& =\frac{1}{2} a^{4} s_{c}^{3}\left(2-B^{*}\right) \int_{\text {sheet }}[\ldots] \frac{P_{0}^{s h}(\tilde{\phi}) s}{|x y|}|\cos \tilde{\phi} \mathrm{d} \tilde{\phi}| \frac{\mathrm{d} s}{(1-A(s))},
\end{aligned}
$$

giving us, since $x y=a^{2} s^{2} n_{1} n_{2}>0$ in the relevant region,

$$
\begin{gathered}
\hat{N}_{\text {sheet }}^{1}=\frac{s_{c}^{3}\left(2-B^{*}\right)}{2} \int_{\text {sheet }}\left(n_{1}^{2}-n_{2}^{2}\right) M(s) \frac{P_{0}^{s h}(\tilde{\phi})}{a} \frac{|\cos \tilde{\phi} \mathrm{d} \tilde{\phi}| \mathrm{d} s}{s(1-A(s))}, \\
\hat{N}_{\text {sheet }}^{2}=\frac{s_{c}^{3}\left(2-B^{*}\right)}{2} \int_{\text {sheet }}\left\{L(s)+\left(n_{2}^{2}-n_{3}^{2}\right) M(s)\right\} \frac{P_{0}^{s h}(\tilde{\phi})}{a} \frac{|\cos \tilde{\phi} \mathrm{d} \tilde{\phi}| \mathrm{d} s}{s(1-A(s))} .
\end{gathered}
$$

The components of $\boldsymbol{n}$ are given by (4.21) and (4.22), and the integrals are carried out numerically.

\subsection{Summary of numerical normal stress results}

At order $c$ in this calculation, the stress is Newtonian (with an enhanced viscosity), and so the normal stress differences are zero to $O(c)$. They appear at order $c^{2}$, because of roughness.

\subsubsection{Comparison with drops}

In the case $\nu=0$ (which is in many ways similar to the case of liquid spheres) the contact probability distribution can be calculated analytically, and is given by

$$
P^{*}=\frac{a s_{c}}{3\left(1-B^{*}\right) \phi^{3}\left(s_{c}\right)}\left\{1-\left[\frac{s_{c}^{2} B^{*}+2\left(1-B^{*}\right) \phi^{2}\left(s_{c}\right) \Psi\left(s_{c}\right)}{s_{c}^{2}\left(B^{*}+2\left(1-B^{*}\right) \sin ^{2} \theta \cos ^{2} \phi\right)}\right]^{3 / 2}\right\} .
$$

The angle integrals may then be carried out analytically, and (after considerable algebra) we obtain

$$
\begin{aligned}
& \hat{N}_{1}=\Phi(2 \mathcal{Y}-10 \mathcal{Z}) M^{*}+\left(\mathcal{Y}-\sin ^{5} \tau\right) I \\
& \hat{N}_{2}=\Phi\left(8 \mathcal{Z} M^{*}-2 \mathcal{Y}\left(L^{*}+M^{*}\right)\right)+\left(4 \sin ^{5} \tau / 5-2 \mathcal{Y}\right) I,
\end{aligned}
$$

where $\hat{N}_{i}=N_{i} / c^{2} \mu|\dot{\gamma}|$,

$$
\begin{aligned}
\mathcal{Y} & =\sin ^{3} \tau / 3+\cos ^{3} \tau(\tau-\tan \tau) \\
\mathcal{Z} & =\left(2-B^{*}\right)\left[\sin ^{5} \tau / 5+\cos ^{3} \tau(3 \tau / 2-\tan \tau-\sin 2 \tau / 4)\right] /\left[6\left(1-B^{*}\right)\right], \\
\sin ^{2} \tau & =2\left(1-B^{*}\right)\left[s_{c}^{2}-\phi^{2}\left(s_{c}\right) \Psi\left(s_{c}\right)\right] /\left[s_{c}^{2}\left(2-B^{*}\right)\right] \\
I & =\frac{4 s_{c}^{5}}{3 \phi^{5}\left(s_{c}\right)}\left(\frac{2-B^{*}}{2\left(1-B^{*}\right)}\right)^{5 / 2} \int_{s=s_{c}}^{\infty} \frac{M(s) \phi^{2}(s) \mathrm{d} s}{s^{3}(1-A(s))}
\end{aligned}
$$

and

$$
\Phi=\frac{2 s_{c}^{3}}{3\left(1-B^{*}\right) \phi^{3}\left(s_{c}\right)}\left(\frac{2-B^{*}}{2\left(1-B^{*}\right)}\right)^{3 / 2}
$$

which matches the results of Zinchenko (1984) in the limit $s_{c} \rightarrow 2$, if the solid sphere mobility functions are substituted into his expression in place of the fluid droplet mobilities. For further verification of the numerical work, a simple program to calculate this result was written and gives results which agree with those from the general code. 


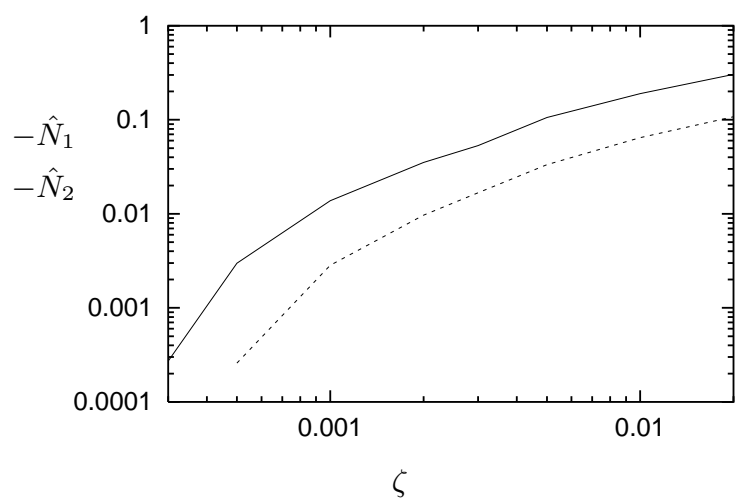

FIGURE 6 . The negative scaled normal stresses plotted against the dimensionless roughness height for $\nu=0$, with the solid and dotted lines representing $-\hat{N}_{1}$ and $-\hat{N}_{2}$ respectively.

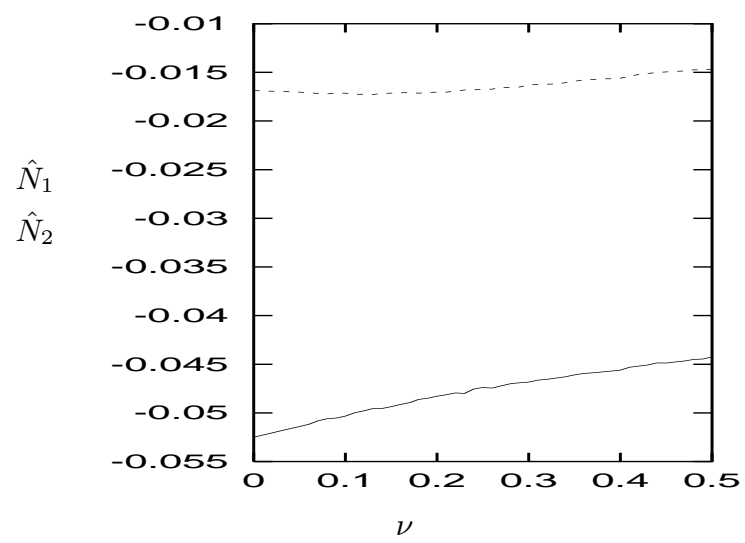

Figure 7 . The dependence of the normal stress differences, $\hat{N}_{1}$ (solid line) and $\hat{N}_{2}$ (dotted line), on the friction coefficient, $\nu$, for a physically plausible value of the roughness height, $\zeta=3 \times 10^{-3}$. Figure modified from original paper.

\subsubsection{Numerical results}

We calculated the two normal stress factors $\hat{N}_{i}$, which depend only on $\zeta$ and $\nu$. Using (4.25), these are

$$
\hat{N}_{i}=\frac{15}{4 \pi}\left(\hat{N}_{\text {bulk }}^{i}+\hat{N}_{\text {contact }}^{i, S}+\hat{N}_{\text {sheet }}^{i}\right)+\frac{9}{16 \pi} \hat{N}_{\text {contact }}^{i, D} .
$$

In figure 6 we plot $\hat{N}_{1}$ and $\hat{N}_{2}$ against $\zeta$ for the slipping or hard-sphere limit $\nu=0$. In each case, both normal stress differences are negative, with $\hat{N}_{1}$ having the larger modulus. Below the critical roughness height $\zeta=2.11 \times 10^{-4}$, no open trajectories intersect with the contact surface, and so no normal stresses are generated. The results show a strong decrease in the normal stresses as $\zeta$ is increased from this critical value.

In figure 7 we plot the two scaled normal stress differences against the friction coefficient, $\nu$, for a typical value of the roughness height, $\zeta=3 \times 10^{-3}$. The dependence of the stresses on $\nu$ is rather weak for both $\hat{N}_{1}$ and $\hat{N}_{2}$; as $\nu$ is increased from 0 to 0.5 (with likely physical values being around $0.1-0.4$ ), the size of $\hat{N}_{1}$ decreases by $15 \%$ and that of $\hat{N}_{2}$ by $13 \%$.

In figure 8 , we take our sample value $\nu=0.3$ and plot the different contributions to 


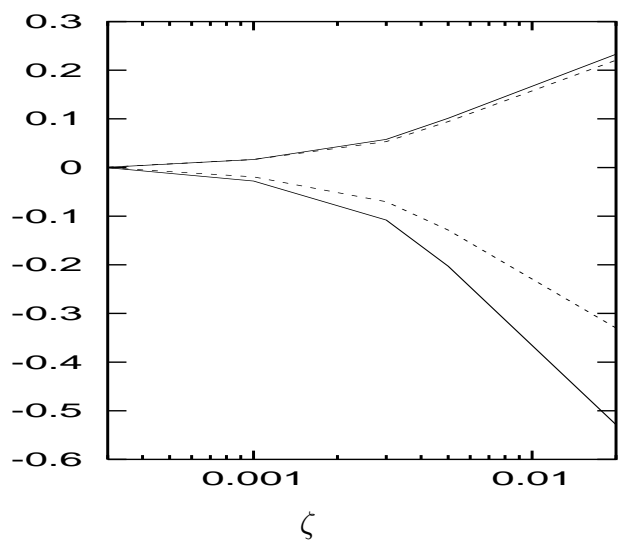

FIGURE 8. The contributions to the scaled normal stresses, plotted against the dimensionless roughness height, with fixed $\nu=0.3$. The solid and dotted lines are for $\hat{N}_{1}$ and $\hat{N}_{2}$ respectively, with the upper curves being the contact contribution to $\hat{N}_{1}$ and the bulk and sheet regions contribution to $\hat{N}_{2}$, and vice versa for the lower curves. Figure modified from original paper.

the normal stresses against $\zeta$. In all cases, the contribution from the bulk is positive, and that from the sheet negative, so we show instead their sum, which indicates which is more important. We observe that there is a competition between the effect of the contact region and that of the rest of the flow. For the first normal stress difference, the majority of the flow causes a negative contribution with the contact contribution smaller and positive, whereas, for the second normal stress difference, the contact contribution is negative and dominates over the smaller, but positive, contribution from the rest of the flow.

\section{Concluding Remarks}

We have investigated the rheology to $O\left(c^{2}\right)$ of a dilute suspension of rough spheres. Because the stress in the suspension is dependent on the flow history, the calculation cannot be carried out for general flows. Instead, we have investigated two steady flows: axisymmetric straining, and shear.

In a steady axisymmetric straining flow, the stress is Newtonian and can be represented by a scalar viscosity. In such flows, the effect of particle contacts due to surface roughness is always to lower the $O\left(c^{2}\right)$ coefficient of the viscosity from the value 6.9 for smooth spheres. The effect of increasing the coefficient of friction is to increase the total viscosity. Although a high coefficient of friction tends to induce rolling (rather than slipping) motion when the particles are in contact, which reduces the lubrication stresses, the rolling causes extra dissipation elsewhere in the flow, and the net effect of adding this additional mechanism of dissipation is to raise the total viscosity relative to frictionless contact. Note that the lowering of the viscosity in a dilute suspension due to particle contact is an effect which we would not expect to see in a concentrated suspension. If many spheres were interacting closely, the interparticle contacts and friction would likely increase the overall viscosity, and possibly cause 'jamming' earlier than would be seen for smooth spheres.

In shear flow, the viscosity cannot be uniquely determined, because of closed orbits of two particles which continue indefinitely unless some other effect is taken into account. However, if the roughness height is above a critical value $\zeta=2.11 \times 10^{-4}$, there are nonzero normal stress differences which may be determined despite the closed orbits. These 
normal stress differences are negative for physical values of the roughness height, with $N_{2}$ slightly smaller in magnitude than $N_{1}$. They depend only weakly on the coefficient of friction, $\nu$.

As the concentration increases, three-particle and even many-particle interactions will become important, and we would expect that the sheet and wake regions might be destroyed. However, the contact regions would be relatively unaffected by the presence of other particles. In straining flows, the viscosity-lowering effect of the excluded volume dominates the remaining contributions (see figure 3) and so the viscosity at moderate concentrations will likely still be lower than that for smooth spheres, though the effect may be less marked. However, at yet higher concentrations when a dilute theory is not applicable, we expect the viscosity to rise as discussed above, so that there is some critical concentration above which our results are not qualitatively useful. In shear, on the other hand, both the bulk and the contact contributions are significant, and so increasing the concentration even a moderate amount would likely have a marked effect on the normal stresses. If the contribution from contact does indeed remain unchanged, then $N_{1}$ will remain negative but smaller in magnitude, whereas it is possible that $N_{2}$ could change sign. However, recent simulations carried out by Foss \& Brady (2000) indicate that both normal stresses remain negative as the concentration increases, so perhaps the dilute asymptotics are more robust than one might suspect.

\section{Acknowledgements}

The authors thank Dr. Alexander Zinchenko for advice on the analysis and Professors Jeffrey Morris and John Brady for helpful discussions, and acknowledge funding from the U.S. National Science Foundation.

\section{REFERENCES}

Arp, P. A. \& Mason, S. G. 1977 The kinetics of flowing dispersions. IX. Doublets of rigid spheres (experimental). J. Colloid Interface Sci. 61, 44-61.

Batchelor, G. K. 1967 An Introduction to Fluid Dynamics. Cambridge University Press.

Batchelor, G. K. \& Green, J. T. $1972 a$ The determination of the bulk stress in a suspension of spherical particles to order $c^{2}$. J. Fluid Mech. 56 (2), 401-427.

Batchelor, G. K. \& Green, J. T. $1972 b$ The hydrodynamic interaction of two small freelymoving spheres in a linear flow field. J. Fluid Mech. 56 (2), 375-400.

Brady, J. F. 1993 Brownian motion, hydrodynamics, and the osmotic pressure. J. Chem. Phys. 98 (4), 3335-3341.

Brady, J. F. \& Morris, J. F. 1997 Microstructure of strongly sheared suspensions and its impact on rheology and diffusion. J. Fluid Mech. 348, 103-139.

DA Cunha, F. R. \& Hinch, E. J. 1996 Shear-induced dispersion in a dilute suspension of rough spheres. J. Fluid Mech. 309, 211-223.

DAvis, R. H. 1992 Effects of surface roughness on a sphere sedimenting through a dilute suspension of neutrally buoyant spheres. Phys. Fluids 4 (12), 2607-2619.

Einstein, A. 1906 Eine neue Bestimmung der Moleküledimensionen. Annalen Phys. 19 (2), $289-306$.

Einstein, A. 1911 Berichtigung zu meiner Arbeit: "Eine neue Bestimmung der Moleküledimensionen". Annalen Phys. 34 (3), 591-592.

Foss, D. R. \& Brady, J. F. 2000 Structure, diffusion and rheology of Brownian suspensions by Stokesian Dynamics simulation. J. Fluid Mech. 400, 1-34.

Kim, S. \& Karrila, S. J. 1991 Microhydrodynamics: Principles and selected applications. Butterworth-Heinemann.

Kim, S. \& Mifflin, R. T. 1985 The resistance and mobility functions of two equal spheres in low-Reynolds-number flow. Phys. Fluids 28 (7), 2033-2045. 
Rampall, I., Smart, J. R. \& Leighton, D. T. 1997 The influence of surface roughness on the particle-pair distribution function of dilute suspensions of non-colloidal spheres in simple shear flow. J. Fluid Mech. 339, 1-24.

Smart, J. R., Beimfohr, S. \& Leighton, D. T. 1993 Measurement of the translational and rotational velocities of a noncolloidal sphere rolling down a smooth inclined plane at low Reynolds number. Phys. Fluids A 5 (1), 13-24.

Smart, J. R. \& Leighton, D. T. 1989 Measurement of the hydrodynamic surface roughness of non-colloidal spheres. Phys. Fluids A 1 (1), 52-60.

Zeng, S., Kerns, E. T. \& DAvis, R. H. 1996 The nature of particle contacts in sedimentation. Phys. Fluids 8 (6), 1389-1396.

Zinchenko, A. Z. 1984 Effect of hydrodynamic interactions between the particles on the rheological properties of dilute emulsions. Prik. Mat. Mekh. 48 (2), 198-206.

\section{Appendix A. Definition of the constants $\beta_{i}$}

We use the definitions of the two-sphere mobility functions given in Kim \& Karrila (1991) to define the constants $\beta_{i}$ as

$$
\begin{aligned}
& \beta_{1}=2 a\left(y_{11}^{a}-y_{12}^{a}\right)-2 a^{2} s_{c}\left(y_{11}^{b}-y_{12}^{b}\right)-\frac{1}{2} a^{3} s_{c}^{2}\left(y_{11}^{c}+y_{12}^{c}\right), \\
& \beta_{2}=s_{c}\left[B^{*}-1+2\left(y_{11}^{h}+y_{12}^{h}\right)\right], \\
& \beta_{3}=2 a\left(x_{11}^{a}-x_{12}^{a}\right), \\
& \beta_{4}=2\left(y_{11}^{h}+y_{12}^{h}\right)+a^{2}\left(\beta_{2} / \beta_{1}\right)\left[\left(y_{11}^{b}-y_{12}^{b}\right)-\frac{1}{2} a s_{c}\left(y_{11}^{c}+y_{12}^{c}\right)\right], \\
& \beta_{5}=s_{c}\left[B^{*}-1+2\left(y_{11}^{h}+y_{12}^{h}\right)\right], \\
& \beta_{6}=\left(s_{c}\left(1-A^{*}\right) / \beta_{3}\right)\left[2 a\left(y_{11}^{a}-y_{12}^{a}\right) / s_{c}+a^{2}\left(y_{11}^{b}-y_{12}^{b}\right)\right] .
\end{aligned}
$$

They are all evaluated at $s=s_{c}$ and specifically defined to be dimensionless, and they are positive in the limit $\zeta \rightarrow 0$.

For the numerical integrations of $\S \S 3$ and 4 , we need numerical forms of the mobilities at all separations. Kim \& Mifflin (1985) presented a collocation method for calculation of the resistance functions for two equal solid spheres in Stokes flow. They have made available their code, along with tables presenting the results at forty equidistant points from $s=2.1$ to $s=6.0$. We used the code to extend the range of these tables. From the resistance functions thus defined, we calculated the mobility functions $A, B, J, K$, $L$ and $M$. In the region $s<2.0025$, the near-field asymptotic forms (from those given in Kim \& Karrila 1991) were used, and in the region $s>6$, the far-field. The function $\phi(s)$ was calculated by numerical integration in the main region from 2.0025 to 6.0 , with the far-field and near-field integrations being performed analytically.

\section{Appendix B. Derivation of the form of the stresslet}

We consider the average stress in a suspension consisting of fluid $(F)$, individual spheres which are force-free $(S)$ and pairs of spheres $(P)$ which exert equal and opposite forces on each other:

$$
\begin{aligned}
\left\langle\sigma_{i j}\right\rangle & =\frac{1}{V} \int_{V_{F}+V_{S}+V_{P}} \sigma_{i j} \mathrm{~d} V \\
& =\frac{1}{V} \int_{V_{F}} \sigma_{i j} \mathrm{~d} V+\frac{1}{V} \sum_{\alpha=1}^{N} \int_{S_{\alpha}} \sigma_{i j} \mathrm{~d} V+\frac{1}{V} \sum_{\beta=1}^{M} \int_{P_{\beta}} \sigma_{i j} \mathrm{~d} V
\end{aligned}
$$

Here $\sigma_{i j}$ is the local stress tensor at a point, and its average $\left\langle\sigma_{i j}\right\rangle$ over the whole volume is the total stresslet which must be averaged over particle configurations to give $\Sigma_{i j}$. 
Note that in a solid, $\sigma_{i j}=\frac{1}{2} \nabla_{k}\left(\sigma_{i k} x_{j}+\sigma_{j k} x_{i}\right)$ and $e_{i j}=0$, so

$$
\begin{aligned}
\left\langle\sigma_{i j}\right\rangle & -\langle p\rangle \delta_{i j}-2 \mu\left\langle e_{i j}\right\rangle \\
= & \frac{1}{V} \sum_{\alpha=1}^{N} \int_{S_{\alpha}} \frac{1}{2} \nabla_{k}\left(\sigma_{i k} x_{j}+\sigma_{j k} x_{i}\right) \mathrm{d} V+\frac{1}{V} \sum_{\beta=1}^{M} \int_{P_{\beta}} \frac{1}{2} \nabla_{k}\left(\sigma_{i k} x_{j}+\sigma_{j k} x_{i}\right) \mathrm{d} V( \\
= & \frac{1}{V} \sum_{\alpha=1}^{N} \int_{S_{\alpha}} \frac{1}{2}\left(\sigma_{i k} x_{j}+\sigma_{j k} x_{i}\right) n_{k} \mathrm{~d} S+\frac{1}{V} \sum_{\beta=1}^{M} \int_{P_{\beta}} \frac{1}{2}\left(\sigma_{i k} x_{j}+\sigma_{j k} x_{i}\right) n_{k} \mathrm{~d} S
\end{aligned}
$$

We then consider one of the pairs $P_{\beta}$. Each sphere is in equilibrium, that is, the net force acting on it is zero. Thus, the hydrodynamic force balances the contact force on each sphere, yielding

$$
\int_{P_{\beta}} \frac{1}{2}\left(\sigma_{i k} x_{j}+\sigma_{j k} x_{i}\right) n_{k} \mathrm{~d} S=\int_{P_{\beta}} \frac{1}{2}\left(\sigma_{i k}^{H} x_{j}+\sigma_{j k}^{H} x_{i}\right) n_{k} \mathrm{~d} S+\int_{P_{\beta}} \frac{1}{2}\left(\sigma_{i k}^{C} x_{j}+\sigma_{j k}^{C} x_{i}\right) n_{k} \mathrm{~d} S,
$$

in which $\sigma^{C}$ is the contribution from the contact force and $\sigma^{H}$ is the hydrodynamic stress balancing it. If the pair $P_{\beta}$ consists of two spheres $S_{\alpha_{m}}$ centred on $\boldsymbol{x}^{\alpha_{m}}$ for $m=1,2$,

$$
\begin{aligned}
\int_{P_{\beta}} \frac{1}{2}\left(\sigma_{i k} x_{j}+\sigma_{j k} x_{i}\right) n_{k} \mathrm{~d} S & =\sum_{m=1}^{2} \int_{S_{\alpha_{m}}} \frac{1}{2}\left(\sigma_{i k}^{H}\left(x_{j}-x_{j}^{\alpha_{m}}\right)+\sigma_{j k}^{H}\left(x_{i}-x_{i}^{\alpha_{m}}\right)\right) n_{k} \mathrm{~d} S \\
+ & \sum_{m=1}^{2} \int_{S_{\alpha_{m}}} \frac{1}{2}\left(\sigma_{i k}^{C}\left(x_{j}-x_{j}^{\alpha_{m}}\right)+\sigma_{j k}^{C}\left(x_{i}-x_{i}^{\alpha_{m}}\right)\right) n_{k} \mathrm{~d} S .
\end{aligned}
$$

Now the stress distribution owing to the contact stress on sphere 1 (which exerts a force $\boldsymbol{F}$ on the fluid at a point $\left.\boldsymbol{x}^{C}=\frac{1}{2}\left(\boldsymbol{x}^{\alpha_{1}}+\boldsymbol{x}^{\alpha_{2}}\right)\right)$ is $\sigma_{i j}^{C} n_{j}=F_{i} \delta\left(\boldsymbol{x}-\boldsymbol{x}^{C}\right)$. A similar form (with the force reversed) holds for sphere 2 , giving

$$
\begin{gathered}
\int_{P_{\beta}} \frac{1}{2}\left(\sigma_{i k} x_{j}+\sigma_{j k} x_{i}\right) n_{k} \mathrm{~d} S=\sum_{m=1}^{2} \int_{S_{\alpha_{m}}} \frac{1}{2}\left(\sigma_{i k}^{H}\left(x_{j}-x_{j}^{\alpha_{m}}\right)+\sigma_{j k}^{H}\left(x_{i}-x_{i}^{\alpha_{m}}\right)\right) n_{k} \mathrm{~d} S \\
+\frac{1}{2}\left[F_{i}\left(x_{j}^{\alpha_{2}}-x_{j}^{\alpha_{1}}\right)+F_{j}\left(x_{i}^{\alpha_{2}}-x_{i}^{\alpha_{1}}\right)\right] .
\end{gathered}
$$

The first of these terms is simply the sum of two stresslets, one for each sphere, centred on the sphere, arising due to the change in motion engendered by the contact force. As such, by the linearity of Stokes flow it is equivalent to the sum of two independent stresslets. The first is the stresslet caused by an equivalent pair of force-free particles in the ambient flow. The second is the stresslet caused by the particles owing to the contact forces and torques acting on them, in a fluid which is otherwise quiescent.

We deduce that the total stresslet generated by a pair of particles at $\mathbf{0}$ and $\boldsymbol{r}$ is given by that in the absence of contact forces (given by Batchelor \& Green 1972a) plus an extra contribution (neglecting isotropic terms) of

$$
D=2 D^{H}+\frac{1}{2}\left(\boldsymbol{F}_{c} \boldsymbol{r}+\boldsymbol{r} \boldsymbol{F}_{c}-2\left(\boldsymbol{F}_{c} \cdot \boldsymbol{r}\right) l\right),
$$

where $\boldsymbol{F}_{c}$ is the force exerted by particle 1 on the fluid, equal and opposite to that exerted by particle 2 , and the term $D^{H}$ is simply the stresslet generated by the forces and torques acting on one of the two spheres in a quiescent fluid. It is multiplied by 2 for the contributions from both particles of the pair. It can be found easily from the mobility formulation for relative motion of two spheres in a quiescent fluid (Kim \& Karrila 1991, 
page 179):

$$
D^{H}=\left(g_{12}-g_{11}\right) \cdot \boldsymbol{F}_{c}+\left(h_{11}+h_{12}\right) \cdot \boldsymbol{T} .
$$

Thus, the contribution from each individual particle, $S^{c}$, is half the deviatoric part of the stresslet given in (B 8), and since $\boldsymbol{T}=-\frac{1}{2} \boldsymbol{F}_{c} \times \boldsymbol{r}$, and $\boldsymbol{r}=a s_{c} \boldsymbol{n}$, after some manipulation we find

$$
\begin{aligned}
S^{c}\left(\boldsymbol{x}_{0}, \boldsymbol{x}_{0}+\boldsymbol{r}\right)=\frac{1}{2} a s[1-A(s)]\left(\boldsymbol{F}_{c} \cdot \boldsymbol{n}\right)\left(\boldsymbol{n n}-\frac{1}{3} l\right) \\
+\frac{1}{4} a s\left[1-B(s)-2\left(y_{11}^{h}+y_{12}^{h}\right)\right]\left(\boldsymbol{F}_{c} \boldsymbol{n}+\boldsymbol{n} \boldsymbol{F}_{c}-2 \boldsymbol{n n}\left(\boldsymbol{F}_{c} \cdot \boldsymbol{n}\right)\right)
\end{aligned}
$$

as in (2.6). 